\title{
A New Double Truncated Generalized Gamma Model with Some Applications
}

\author{
Awad A. Bakery $\mathbb{D}^{1,2}$ Wael Zakaria $\mathbb{D}^{1}{ }^{2}$ and OM Kalthum S. K. Mohamed $\mathbb{D}^{1,3}$ \\ ${ }^{1}$ University of Jeddah, College of Science and Arts at Khulis, Department of Mathematics, Jeddah, Saudi Arabia \\ ${ }^{2}$ Department of Mathematics, Faculty of Science, Ain Shams University, P.O. Box 1156, Abbassia, Cairo 11566, Egypt \\ ${ }^{3}$ Academy of Engineering and Medical Sciences, Department of Mathematics, Khartoum, Sudan
}

Correspondence should be addressed to OM Kalthum S. K. Mohamed; om_kalsoom2020@yahoo.com

Received 7 May 2021; Accepted 6 August 2021; Published 17 August 2021

Academic Editor: Ghulam Mustafa

Copyright (c) 2021 Awad A. Bakery et al. This is an open access article distributed under the Creative Commons Attribution License, which permits unrestricted use, distribution, and reproduction in any medium, provided the original work is properly cited.

\begin{abstract}
The generalized Gamma model has been applied in a variety of research fields, including reliability engineering and lifetime analysis. Indeed, we know that, from the above, it is unbounded. Data have a bounded service area in a variety of applications. A new five-parameter bounded generalized Gamma model, the bounded Weibull model with four parameters, the bounded Gamma model with four parameters, the bounded generalized Gaussian model with three parameters, the bounded exponential model with three parameters, and the bounded Rayleigh model with two parameters, is presented in this paper as a special case. This approach to the problem, which utilizes a bounded support area, allows for a great deal of versatility in fitting various shapes of observed data. Numerous properties of the proposed distribution have been deduced, including explicit expressions for the moments, quantiles, mode, moment generating function, mean variance, mean residual lifespan, and entropies, skewness, kurtosis, hazard function, survival function, $r$ th order statistic, and median distributions. The delivery has hazard frequencies that are monotonically increasing or declining, bathtub-shaped, or upside-down bathtub-shaped. We use the Newton Raphson approach to approximate model parameters that increase the log-likelihood function and some of the parameters have a closed iterative structure. Six actual data sets and six simulated data sets were tested to demonstrate how the proposed model works in reality. We illustrate why the Model is more stable and less affected by sample size. Additionally, the suggested model for wavelet histogram fitting of images and sounds is very accurate.
\end{abstract}

\section{Introduction}

The gamma (ГМ) model, including Weibull, gamma, exponential, and Rayleigh as special submodels, among others, is a very popular distribution for modeling lifetime data and for modeling phenomenon with monotone failure rates. An advantage of $\Gamma M$ is that it requires a little measure of parameters for learning. Also, these parameters can be measured by getting the expectation maximization (EM) algorithm $[1,2]$ to maximize the log-likelihood function. The early generalization of gamma distribution can be traced back to Amoroso [3] who discussed a generalized gamma distribution and applied it to fit income rates. Johnson et al. [4] gave a four parameter generalized gamma distribution which reduces to the generalized gamma distribution defined by Stacy [2] when the location parameter is set to zero. Mudholkar and Srivastava [5] introduced the exponentiated method to derive a distribution. The generalized gamma defined by Stacy [2] is a three-parameter exponentiated gamma distribution. Agarwal and Al-Saleh [6] applied generalized gamma to study hazard rates. Balakrishnan and Peng [7] applied this distribution to develop generalized gamma frailty model. Cordeiro et al. [8] derived another generalization of Stacys generalized gamma distribution using exponentiated method and applied it to life time and survival analysis. Nadarajah and Gupta [9] proposed another type of generalized gamma distribution with application to fit drought 
data. As of late, Chen et al. [10] used generalized gamma distribution with three parameters for flood frequency analysis, Zhao et al. [11] used generalized gamma distribution with three parameters to give the statistical characterizes of high-resolution SAR images, and Mead et al. [12] defined modified generalized gamma distribution so as to investigate greater flexibility in modeling data from a practical viewpoint and they derived multifarious identities and properties of this distribution, including explicit expressions for the moments, quantiles, mode, moment generating function, mean deviation, mean residual lifetime, and expression of the entropies. We extend all the past models with five parameters to range $\mathbb{R}$ (real numbers) or any bounded subset of $\mathbb{R}$. Fulger et al. [13] generate random numbers within any arbitrary interval. We introduce in this paper the high flexibility of a bounded generalized Gamma model with five parameters (BGГM) for analyzing data. The BGГM Model is of noticeable significance for image coding, compression applications, sound system, wind speed data, and breast cancer data fitting. This new distribution has a flexibility to fit any kind of observed data whose pdf is monotonically increasing, decreasing, bathtub, and upside down bathtub-shaped depending on the parameter values and bounded support regions. The remainder of this paper is organized as follows: The ВGГM with its sub models and some shapes describe the hazard rate function are defined in Section 2. Some properties of the ВGГM distribution are studied in Section 3 including, quantile, mode, moments, moment generating function, mean deviation, mean residual life and entropy. Section 4 presents the parameter estimation. Section 5 sets out the experimental results. Section 6 presents our conclusions.

\section{The Bounded Generalized Gamma Model and Its Special Models}

The standard form of gamma function is

$$
\Gamma(\eta)=\int_{0}^{\infty} x^{\eta-1} e^{-x} \mathrm{~d} x, \quad \eta>0 .
$$

The incomplete gamma function is defined by

$$
\Gamma(\eta, s)=\frac{1}{\Gamma(\eta)} \int_{0}^{s} x^{\eta-1} e^{-x} \mathrm{~d} x, \quad \eta>0 \text { and } s \geq 0 .
$$

The probability density function (pdf) of the generalized gamma distribution is given by

$$
T(x \mid \Theta)=\frac{\lambda \beta^{\eta}}{2 \delta \Gamma(\eta)}\left(\frac{|x-u|}{\delta}\right)^{\eta \lambda-1} e^{-\beta(|x-u| / \delta)^{\lambda}},
$$

for all $x \in \mathbb{R}$, where $\Theta=(u, \delta, \beta, \eta, \lambda)^{\prime}, \delta, \eta, \lambda, \beta>0$ and $u \in \mathbb{R}$. The cumulative distribution function (cdf) of generalized gamma distribution defined as follows:

$$
\begin{aligned}
D(z) & =\int_{-\infty}^{z} T(x \mid \Theta) \mathrm{d} x=\int_{-\infty}^{z} \frac{\lambda \beta^{\eta}}{2 \delta \Gamma(\eta)}\left(\frac{|x-u|}{\delta}\right)^{\eta \lambda-1} e^{-\beta(|x-u| \mid \delta)^{\lambda}} \mathrm{d} x \\
& =\frac{1}{2}+\frac{1}{2} \operatorname{sign}(z-u)\left[\Gamma\left(\eta, \beta\left(\frac{|z-u|}{\delta}\right)^{\lambda}\right)-\Gamma\left(\eta, \beta\left(\frac{|u|}{\delta}\right)^{\lambda}\right)\right] .
\end{aligned}
$$
by

Let $\Omega=[a, b] \subseteq \mathbb{R}$ and we denote the indicator function

$$
1_{\Omega}(x)= \begin{cases}1, & \text { if } x \in \Omega, \\ 0, & \text { if otherwise. }\end{cases}
$$

We define the pdf of the bounded generalized gamma distribution (ВGГM) as

$$
\begin{aligned}
\Upsilon & =\Upsilon(x \mid \Theta)=\frac{T(x \mid \Theta)}{\int_{\Omega} T(x \mid \Theta) \mathrm{d} x} \\
& =\frac{\lambda \beta^{\eta} / 2 \delta \Gamma(\eta)(|x-u| / \delta)^{\eta \lambda-1} e^{-\beta(|x-u| / \delta)^{\lambda}}}{\int_{\Omega} T(x \mid \Theta) \mathrm{d} x}, \text { for all } x \in \Omega .
\end{aligned}
$$

In another form, we can write the pdf of the bounded generalized gamma distribution (ВGГM) as

$$
\Upsilon=\frac{\lambda \beta^{\eta} / 2 \delta \Gamma(\eta)(|x-u| / \delta)^{\eta \lambda-1} e^{-\beta(|x-u| / \delta)^{\lambda}}}{D(b)-D(a)},
$$

where

$$
\begin{aligned}
D(b)-D(a)= & \frac{1}{2} \operatorname{sign}(b-u)\left[\Gamma\left(\eta, \beta\left(\frac{|b-u|}{\delta}\right)^{\lambda}\right)-\Gamma\left(\eta, \beta\left(\frac{|u|}{\delta}\right)^{\lambda}\right)\right] \\
& -\frac{1}{2} \operatorname{sign}(a-u)\left[\Gamma\left(\eta, \beta\left(\frac{|a-u|}{\delta}\right)^{\lambda}\right)-\Gamma\left(\eta, \beta\left(\frac{|u|}{\delta}\right)^{\lambda}\right)\right] .
\end{aligned}
$$

It is clear to see that

$$
\Upsilon \geq 0 \text { and } \int_{\Omega} \Upsilon(x \mid u, \delta, \beta, \eta, \lambda) \mathrm{d} x=1 .
$$

Hence, the cdf of the bounded generalized gamma distribution (ВGГМ) is given by

$$
\begin{aligned}
\Phi(x) & =\frac{D(x)-D(a)}{D(b)-D(a)} \\
& =\frac{\operatorname{sign}(x-u)\left[\Gamma\left(\eta, \beta(|x-u| / \delta)^{\lambda}\right)-\Gamma\left(\eta, \beta(|u| / \delta)^{\lambda}\right)\right]-\operatorname{sign}(a-u)\left[\Gamma\left(\eta, \beta(|a-u| / \delta)^{\lambda}\right)-\Gamma\left(\eta, \beta(|u| / \delta)^{\lambda}\right)\right]}{\operatorname{sign}(b-u)\left[\Gamma\left(\eta, \beta(|b-u| / \delta)^{\lambda}\right)-\Gamma\left(\eta, \beta(|u| / \delta)^{\lambda}\right)\right]-\operatorname{sign}(a-u)\left[\Gamma\left(\eta, \beta(|a-u| / \delta)^{\lambda}\right)-\Gamma\left(\eta, \beta(|u| / \delta)^{\lambda}\right)\right]} .
\end{aligned}
$$


The parameters $u(\delta, \beta)$ and $(\eta, \lambda)$ are corresponding to the location, scale, and shape parameters, respectively. Note that $\Upsilon(x \mid \Theta)$ can be any kind of distribution, for example, in exponential distribution (ED) $[14,15]$ be $\phi(x \mid u, \delta, \beta)$, Weibull distribution (WD) [16-18] be $T(x \mid u, \delta, \beta, \lambda)$, Rayleigh distribution (RD) $[19,20]$ be $T(x \mid u, \delta)$, generalized Gaussian distribution (GGD) [21] be $T(x \mid u, \delta, \lambda)$, Gaussian distribution (GD) [15] be $T(x \mid u, \delta)$, Laplacian distribution (LD) [22] be $T(x \mid u, \delta)$ and Gamma distribution (ГD) [1] be $T(x \mid u, \delta, \eta, \beta)$. These distributions are all unbounded with support range $(0, \infty)$. We extend all the past models with range $(-\infty, \infty)$ also to the bounded case. The ВGГM has several models as special cases, which makes it distinguishable scientific importance from other models. We investigate the various special models of the ВGГM as listed in Table 1. The survival function and hazard rate function for BGГM are, respectively, given by
TABLE 1: The comparative models are special cases of the ВGГM.

\begin{tabular}{|c|c|}
\hline GГМ & ВGГM: $\Omega=\mathbb{R}$ \\
\hline ВГМ & ВGГМ: $\lambda=1$ \\
\hline$\Gamma \mathrm{M}$ & ВGГM: $\Omega=\mathbb{R}$ and $\lambda=1$ \\
\hline BWM & ВGГМ: $\eta=1$ \\
\hline WM & ВGГМ: $\Omega=\mathbb{R}, \eta=1$ \\
\hline BGGM & BGГM: $\eta=1 / \lambda$ and $\beta=(\Gamma(3 / \lambda) / \Gamma(1 / \lambda))^{\lambda / 2}$ \\
\hline GGM & ВGГM: $\Omega=\mathbb{R}, \eta=1 / \lambda$ and $\beta=(\Gamma(3 / \lambda) / \Gamma(1 / \lambda))^{\lambda / 2}$ \\
\hline BEM & BGГM: $\lambda=1$, and $\eta=1$ \\
\hline EM & ВGГM: $\Omega=\mathbb{R}, \lambda=1$, and $\eta=1$ \\
\hline BGM & ВGГM: $\lambda=2, \eta=0.5$ and $\beta=0.5$ \\
\hline GM & ВGГM: $\Omega=\mathbb{R}, \lambda=2, \eta=0.5$ and $\beta=0.5$ \\
\hline BRM & ВGГM: $\lambda=2, \eta=1$ and $\beta=0.5$ \\
\hline $\mathrm{RM}$ & ВGГM: $\Omega=\mathbb{R}, \lambda=2, \eta=1$ and $\beta=0.5$ \\
\hline BLM & ВGГM: $\lambda=1, \eta=1$ and $\beta=\sqrt{2}$ \\
\hline LM & ВGГМ: $\Omega=\mathbb{R}, \lambda=1, \eta=1$ and $\beta=\sqrt{2}$ \\
\hline
\end{tabular}

$$
\begin{aligned}
S(x) & =1-\Phi(x) \\
& =\frac{\operatorname{sign}(b-u)\left[\Gamma\left(\eta, \beta(|b-u| / \delta)^{\lambda}\right)-\Gamma\left(\eta, \beta(|u| / \delta)^{\lambda}\right)\right]-\operatorname{sign}(x-u)\left[\Gamma\left(\eta, \beta(|x-u| / \delta)^{\lambda}\right)-\Gamma\left(\eta, \beta(|u| / \delta)^{\lambda}\right)\right]}{\operatorname{sign}(b-u)\left[\Gamma\left(\eta, \beta(|b-u| / \delta)^{\lambda}\right)-\Gamma\left(\eta, \beta(|u| / \delta)^{\lambda}\right)\right]-\operatorname{sign}(a-u)\left[\Gamma\left(\eta, \beta(|a-u| / \delta)^{\lambda}\right)-\Gamma\left(\eta, \beta(|u| / \delta)^{\lambda}\right)\right]^{\prime}}, \\
\wedge(x) & =\frac{\Upsilon(x \mid \Theta)}{S(x)} \\
& =\frac{\lambda \beta^{\eta} / 2 \delta \Gamma(\eta)(|x-u| / \delta)^{\eta \lambda-1} e^{-\beta(|x-u| / \delta)^{\lambda}}}{\operatorname{sign}(b-u)\left[\Gamma\left(\eta, \beta(|b-u| / \delta)^{\lambda}\right)-\Gamma\left(\eta, \beta(|u| / \delta)^{\lambda}\right)\right]-\operatorname{sign}(x-u)\left[\Gamma\left(\eta, \beta(|x-u| / \delta)^{\lambda}\right)-\Gamma\left(\eta, \beta(|u| / \delta)^{\lambda}\right)\right]} .
\end{aligned}
$$

In Figures 1 and 2, we display the plots of the pdf of ВGГM for various parameters. Figure 3 displays the ВGГM failure rate function which can be increasing, decreasing, bathtub, and upside down bathtub-shaped depending on the parameter values.

\section{Properties of BGГM}

In this section, we provide some general properties of the ВGГM including quantile function, mode, moments, mean deviation, mean residual life and mean waiting time, Rényi entropy, and order statistics.

3.1. Mode and Quantile. The $p^{\text {th }}$ quantile function of the $\mathrm{B} G \Gamma \mathrm{M}$ is the solution of

$$
\begin{aligned}
\Phi\left(x_{p}\right)= & \frac{D\left(x_{p}\right)-D(a)}{D(b)-D(a)}=p \Rightarrow \\
& \frac{\operatorname{sign}\left(x_{p}-u\right)\left[\Gamma\left(\eta, \beta\left(\left|x_{p}-u\right| / \delta\right)^{\lambda}\right)-\Gamma\left(\eta, \beta(|u| / \delta)^{\lambda}\right)\right]-\operatorname{sign}(a-u)\left[\Gamma\left(\eta, \beta(|a-u| / \delta)^{\lambda}\right)-\Gamma\left(\eta, \beta(|u| / \delta)^{\lambda}\right)\right]}{\operatorname{sign}(b-u)\left[\Gamma\left(\eta, \beta(|b-u| / \delta)^{\lambda}\right)-\Gamma\left(\eta, \beta(|u| / \delta)^{\lambda}\right)\right]-\operatorname{sign}(a-u)\left[\Gamma\left(\eta, \beta(|a-u| / \delta)^{\lambda}\right)-\Gamma\left(\eta, \beta(|u| / \delta)^{\lambda}\right)\right]}=p .
\end{aligned}
$$

The median, denoted by $\mu^{*}$, can be obtained by substituting $p=0.5$ in 10 and solving the equation 


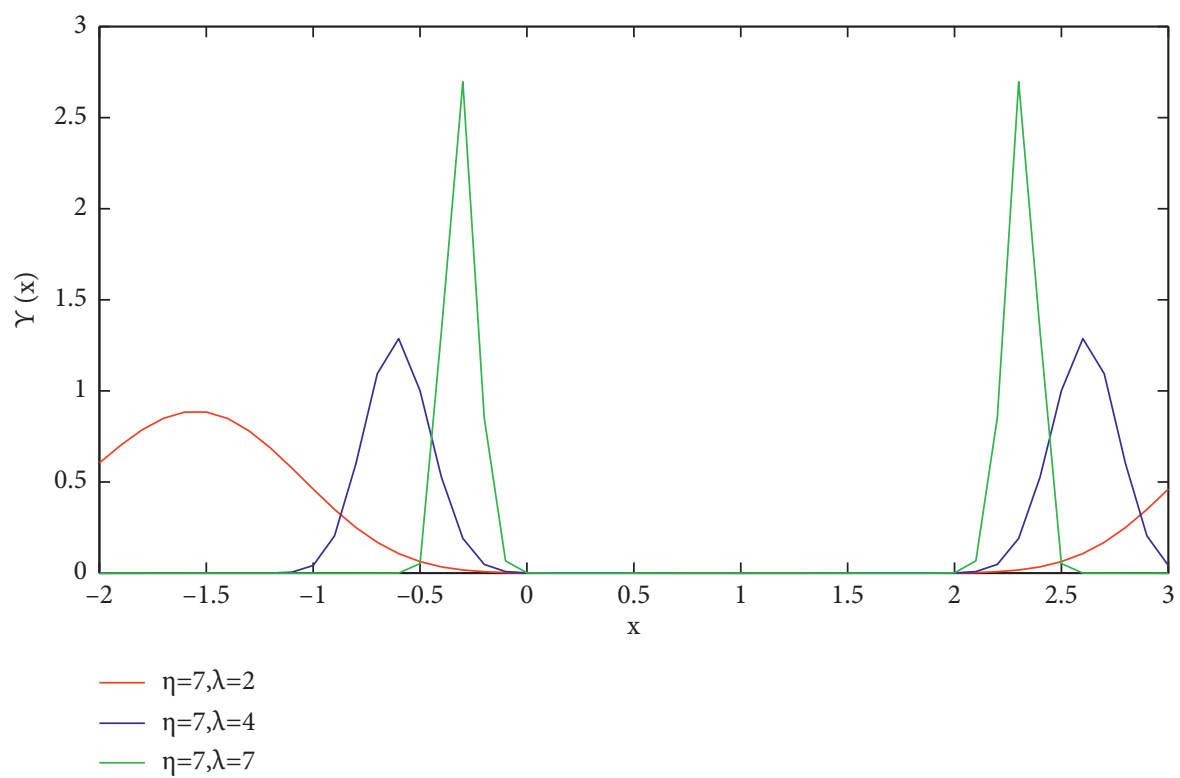

(a)

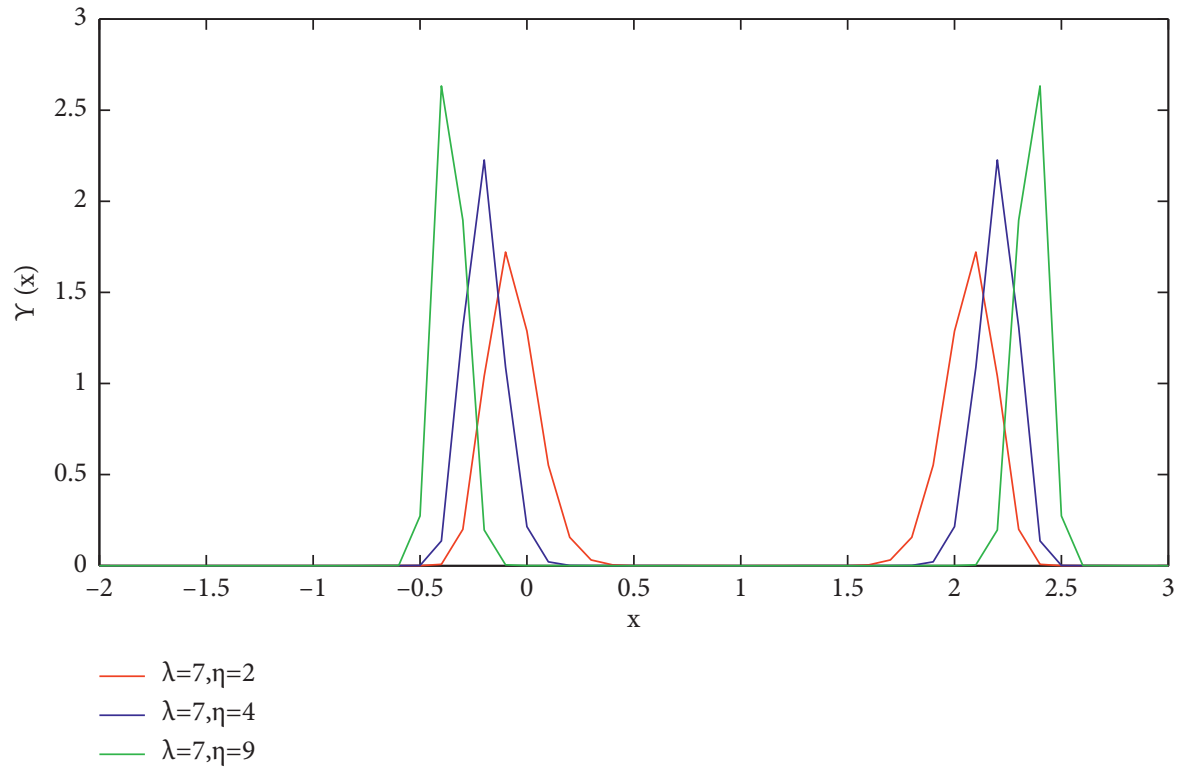

(b)

Figure 1: Continued. 


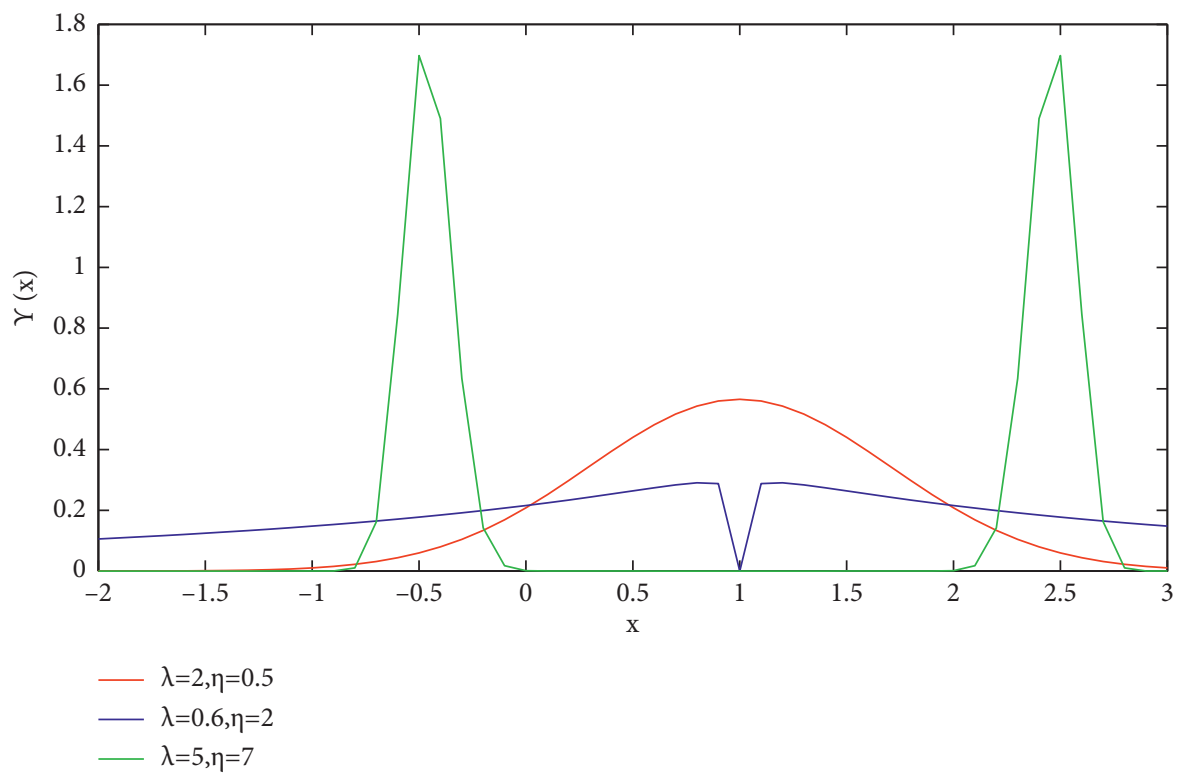

(c)

FigURE 1: The pdf of BGГM for $a=-2, b=3, u=1, \delta=1, \beta=1$ and (a) $\eta=7$ and $\lambda=2,4,7$; (b) $\lambda=7$ and $\eta=2,4,9$; (c) $(\eta=0.5, \lambda=2)$, $(\eta=2, \lambda=0.6)$ and $(\eta=7, \lambda=5)$.

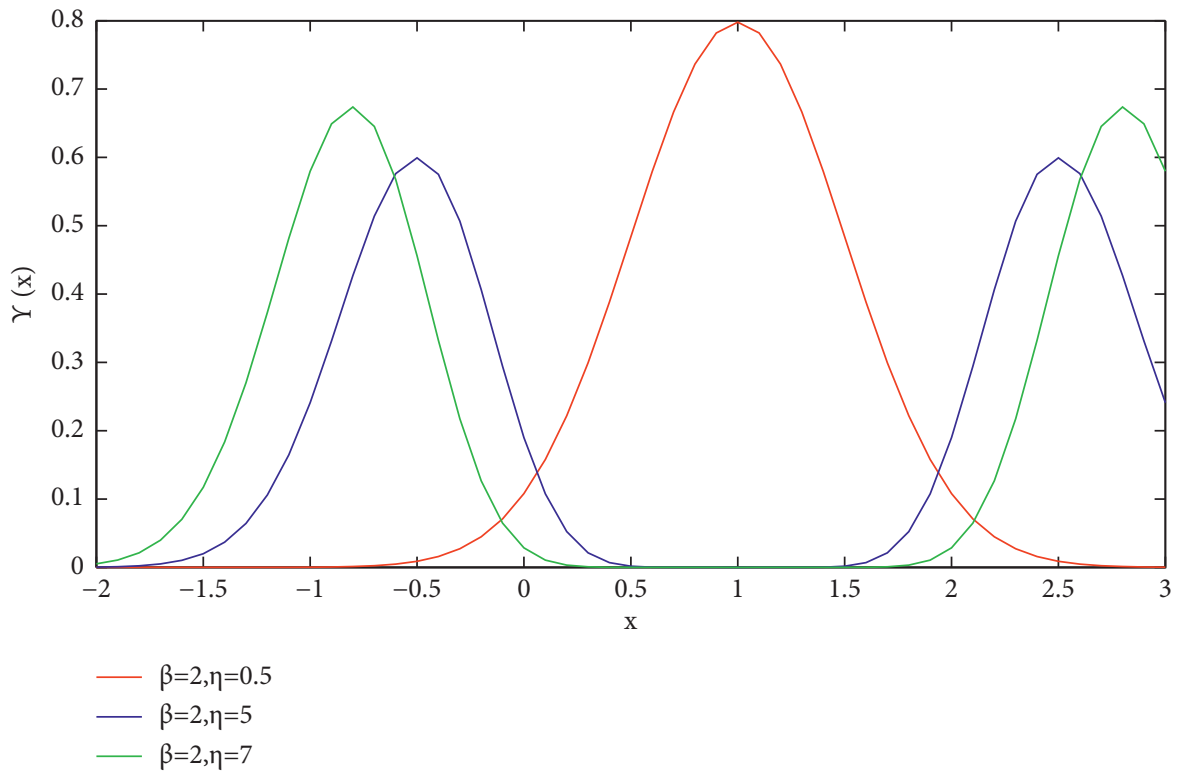

(a)

Figure 2: Continued. 


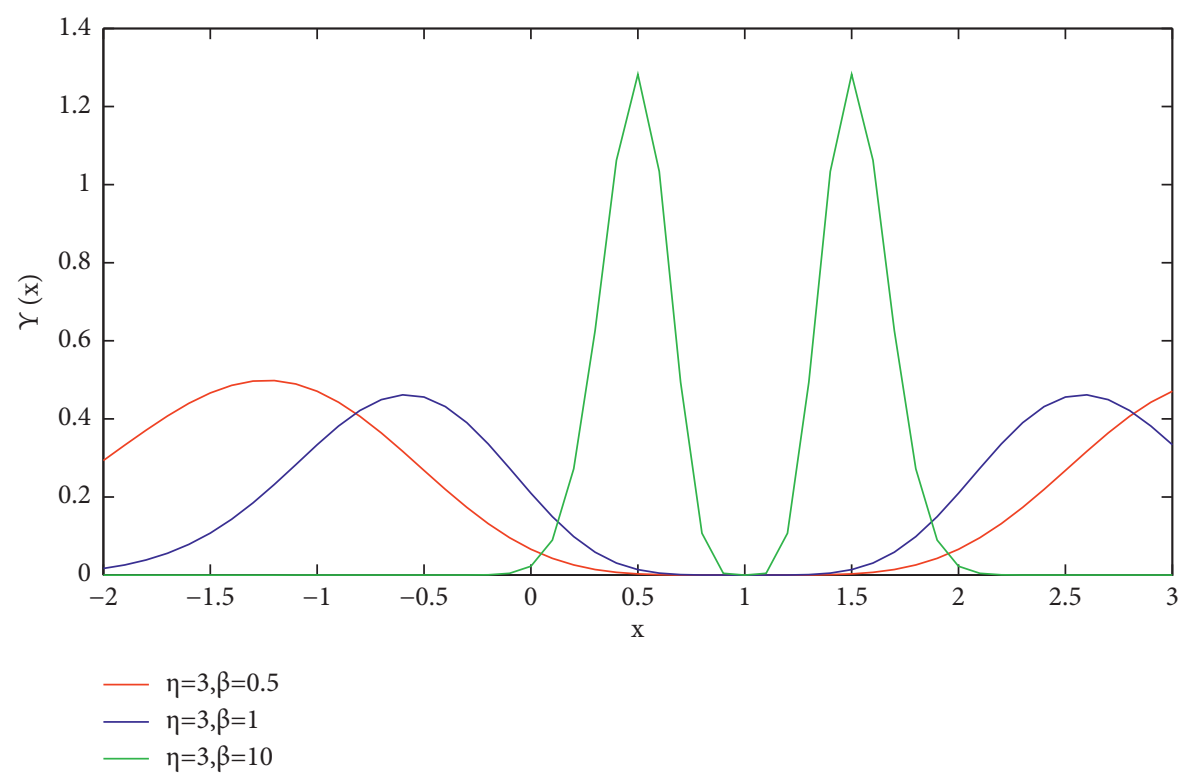

(b)

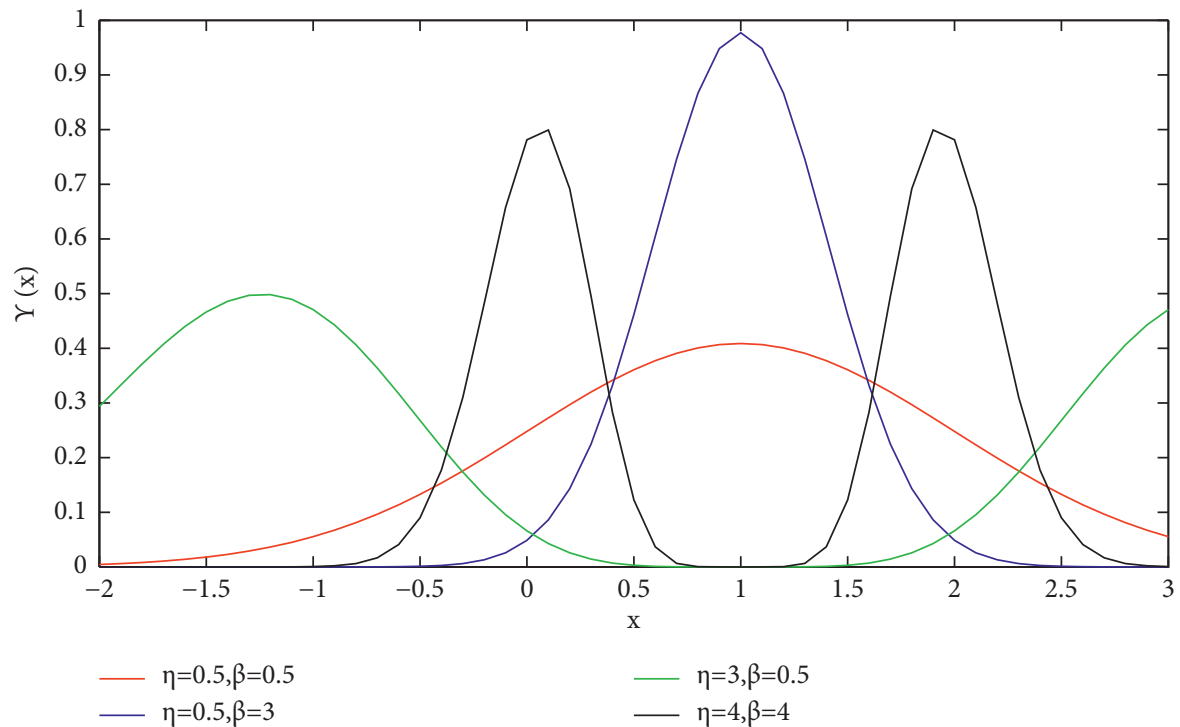

(c)

Figure 2: The pdf of BGГM for $a=-2, b=3, u=1, \delta=1, \lambda=2$ and (a) $\beta=2$ and $\eta=0.5,5,7$; (b) $\eta=3$ and $\beta=0.5,1,10$; (c) $(\eta=0.5, \beta=0.5),(\eta=0.5, \beta=3),(\eta=3, \beta=0.5)$ and $(\eta=4, \beta=4)$.

$$
\frac{\operatorname{sign}\left(\mu^{*}-u\right)\left[\Gamma\left(\eta, \beta\left(\left|\mu^{*}-u\right| / \delta\right)^{\lambda}\right)-\Gamma\left(\eta, \beta(|u| / \delta)^{\lambda}\right)\right]-\operatorname{sign}(a-u)\left[\Gamma\left(\eta, \beta(|a-u| / \delta)^{\lambda}\right)-\Gamma\left(\eta, \beta(|u| / \delta)^{\lambda}\right)\right]}{\operatorname{sign}(b-u)\left[\Gamma\left(\eta, \beta(|b-u| / \delta)^{\lambda}\right)-\Gamma\left(\eta, \beta(|u| / \delta)^{\lambda}\right)\right]-\operatorname{sign}(a-u)\left[\Gamma\left(\eta, \beta(|a-u| / \delta)^{\lambda}\right)-\Gamma\left(\eta, \beta(|u| / \delta)^{\lambda}\right)\right]}=0.5 .
$$

The mode, denoted by $x_{m}$ of the $\mathrm{B} G \Gamma$ distribution, is given by

$$
x_{m}=u \pm \delta\left(\frac{\eta \lambda-1}{\beta \lambda}\right)^{1 / \lambda}, \text { such that } \Upsilon^{\prime \prime}\left(x_{m} \mid \Theta\right)<0
$$

\section{Remark 1}

(1) If $\eta \lambda=1$, then the BGГ distribution is unimodal distribution

(2) If $\eta \lambda>1$, then the BGГ distribution is multimodal distribution 


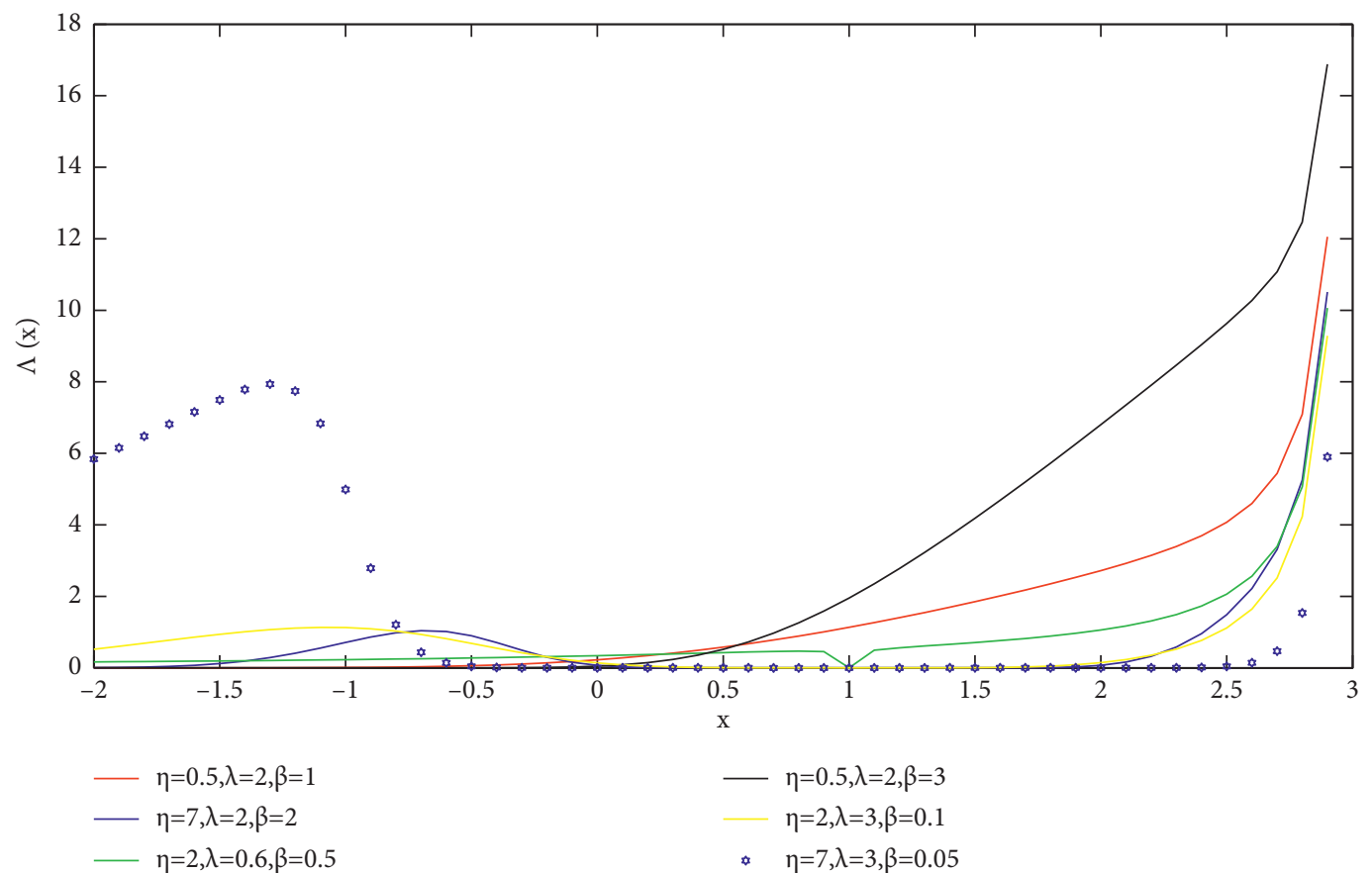

Figure 3: The hazard plots of ВGГM for $a=-2, b=3, u=1, \delta=1$ and different values of $\beta, \eta$ and $\lambda$.

3.2. Moments, Generating Function, and Mean Deviation. The $r^{\text {th }}$ moment about zero of BGГ distribution is

$$
\begin{aligned}
E\left(x^{r}\right) & =\int_{a}^{b} x^{r} \Upsilon(x \mid \Theta) \mathrm{d} x \\
& =\frac{ \pm \sum_{n=0}^{r}\left(\begin{array}{c}
r \\
n
\end{array}\right) u^{n}\left( \pm \delta / \beta^{1 / \lambda}\right)^{r-n} \Gamma(\eta+(r-n / \lambda))\left[\Gamma\left(\eta+(r-n / \lambda), \beta(|b-u| / \delta)^{\lambda}\right)-\Gamma\left(\eta+(r-n / \lambda), \beta(|a-u| / \delta)^{\lambda}\right)\right]}{2 \Gamma(\eta)(D(b)-D(a))} .
\end{aligned}
$$

The mean $\mu$ of the $B G \Gamma$ distribution is given by

$$
\begin{aligned}
\mu= & E(x) \\
= & \frac{ \pm 1}{2 \Gamma(\eta)(D(b)-D(a))} \pm \delta \beta^{1 / \lambda} \Gamma\left(\eta+\frac{1}{\lambda}\right)\left[\Gamma\left(\eta+\frac{1}{\lambda}, \beta\left(\frac{|b-u|}{\delta}\right)^{\lambda}\right)-\Gamma\left(\eta+\frac{1}{\lambda}, \beta\left(\frac{|a-u|}{\delta}\right)^{\lambda}\right)\right] \\
& +u \Gamma(\eta)\left[\Gamma\left(\eta, \beta\left(\frac{|b-u|}{\delta}\right)^{\lambda}\right)-\Gamma\left(\eta, \beta\left(\frac{|a-u|}{\delta}\right)^{\lambda}\right)\right] .
\end{aligned}
$$

The variance $\sigma^{2}$ of the ВGГ distribution is given by 


$$
\begin{aligned}
& \sigma^{2}=E\left(x^{2}\right)-E^{2}(x) \\
& =\frac{ \pm 1}{2 \Gamma(\eta)(D(b)-D(a))}\left\{\frac{\delta^{2}}{\beta^{2 / \lambda}} \Gamma\left(\eta+\frac{2}{\lambda}\right)\left[\Gamma\left(\eta+\frac{2}{\lambda}, \beta\left(\frac{|b-u|}{\delta}\right)^{\lambda}\right)-\Gamma\left(\eta+\frac{2}{\lambda}, \beta\left(\frac{|a-u|}{\delta}\right)^{\lambda}\right)\right]\right. \\
& \pm \frac{2 u \delta}{\beta^{1 / \lambda}} \Gamma\left(\eta+\frac{1}{\lambda}\right)\left[\Gamma\left(\eta+\frac{1}{\lambda}, \beta\left(\frac{|b-u|}{\delta}\right)^{\lambda}\right)\right. \\
& \left.-\Gamma\left(\eta+\frac{1}{\lambda}, \beta\left(\frac{|a-u|}{\delta}\right)^{\lambda}\right)\right] \\
& \left.+u^{2} \Gamma(\eta)\left[\Gamma\left(\eta, \beta\left(\frac{|b-u|}{\delta}\right)^{\lambda}\right)-\Gamma\left(\eta, \beta\left(\frac{|a-u|}{\delta}\right)^{\lambda}\right)\right]\right\}-\mu^{2}
\end{aligned}
$$

The central moments of BGГ distribution can be obtained as follows

$$
\begin{aligned}
E\left((x-\mu)^{r}\right)= & \int_{a}^{b}(x-\mu)^{r} \Upsilon(x \mid \Theta) \mathrm{d} x \\
= & \frac{ \pm 1}{2 \Gamma(\eta)(D(b)-D(a))} \sum_{n=0}^{r}\left(\begin{array}{c}
r \\
n
\end{array}\right) u^{n}\left(\frac{ \pm \delta}{\beta^{1 / \lambda}}\right)^{r-n} \Gamma\left(\eta+\frac{r-n}{\lambda}\right)\left[\Gamma\left(\eta+\frac{r-n}{\lambda}, \beta\left(\frac{|b-u|}{\delta}\right)^{\lambda}\right)\right. \\
& \left.-\Gamma\left(\eta+\frac{r-n}{\lambda}, \beta\left(\frac{|a-u|}{\delta}\right)^{\lambda}\right)\right] \sum_{j=0}^{r}\left(\begin{array}{c}
r \\
j
\end{array}\right)(-\mu)^{r-j} \\
\gamma_{1}= & \frac{E\left((x-\mu)^{3}\right)}{\sigma^{3}}, \\
\gamma_{2}= & \frac{E\left((x-\mu)^{4}\right)}{\sigma^{4}} .
\end{aligned}
$$

The moment generating function $M_{x}(t)$ of BGГ distribution is

$$
\begin{aligned}
& M_{x}(t)= \int_{a}^{b} e^{t x} \Upsilon(x \mid \Theta) \mathrm{d} x \\
& \\
&= \pm \sum_{r=0}^{\infty} t^{r} / r ! \sum_{n=0}^{r}\left(\begin{array}{c}
r \\
n
\end{array}\right) u^{n}\left( \pm \delta / \beta^{1 / \lambda}\right)^{r-n} \Gamma(\eta+(r-n / \lambda))\left[\Gamma\left(\eta+(r-n / \lambda), \beta(|b-u| / \delta)^{\lambda}\right)-\Gamma\left(\eta+(r-n / \lambda), \beta(|a-u| / \delta)^{\lambda}\right)\right] \\
& 2 \Gamma(\eta)(D(b)-D(a))
\end{aligned}
$$

The mean deviation $\mathrm{Md}$ of $\mathrm{BG \Gamma}$ distribution can be derived as 


$$
\begin{aligned}
\operatorname{Md}= & E(|x-\mu|)=\int_{a}^{b}|x-\mu| \Upsilon(x \mid \Theta) \mathrm{d} x=2\left[\mu \Phi(\mu)-\int_{a}^{\mu} x \Upsilon(x \mid \Theta) \mathrm{d} x\right] \\
= & \frac{2 \mu \operatorname{sign}(\mu-u)\left[\Gamma\left(\eta, \beta(|\mu-u| / \delta)^{\lambda}\right)-\Gamma\left(\eta, \beta(|u| / \delta)^{\lambda}\right)\right]-\operatorname{sign}(a-u)\left[\Gamma\left(\eta, \beta(|a-u| / \delta)^{\lambda}\right)-\Gamma\left(\eta, \beta(|u| / \delta)^{\lambda}\right)\right]}{\operatorname{sign}(b-u)\left[\Gamma\left(\eta, \beta(|b-u| / \delta)^{\lambda}\right)-\Gamma\left(\eta, \beta(|u| / \delta)^{\lambda}\right)\right]-\operatorname{sign}(a-u)\left[\Gamma\left(\eta, \beta(|a-u| / \delta)^{\lambda}\right)-\Gamma\left(\eta, \beta(|u| / \delta)^{\lambda}\right)\right]} \\
& -\frac{1}{\Gamma(\eta)(D(b)-D(a))} \frac{\delta}{\beta^{1 / \lambda}} \Gamma\left(\eta+\frac{1}{\lambda}\right)\left[\Gamma\left(\eta+\frac{1}{\lambda}, \beta\left(\frac{|\mu-u|}{\delta}\right)^{\lambda}\right)-\Gamma\left(\eta+\frac{1}{\lambda}, \beta\left(\frac{|a-u|}{\delta}\right)^{\lambda}\right)\right] \\
& \Gamma(\eta)\left[\Gamma\left(\eta, \beta\left(\frac{|\mu-u|}{\delta}\right)^{\lambda}\right)-\Gamma\left(\eta, \beta\left(\frac{|a-u|}{\delta}\right)^{\lambda}\right)\right] .
\end{aligned}
$$

In Table 2, the Median, Mode, Mean, Variance, Skewness, and Kurtosis of BGГM have given for $a=-2, b=3$, $u=1, \delta=1$, and $\beta=1$ and various values of $\eta$ and $\lambda$. From Table 2 , we note that for fixed values of $a, b, u, \delta, \beta$, and $\eta$, the Kurtosis is decreasing function of $\lambda$. Also, for fixed values of $a, b, u, \delta, \beta$, and $\lambda$, the Mode 1 , Variance, and Skewness are increasing function and the Mode 2 and Mean are decreasing function of $\eta$. In Table 3, Median, Mode, Mean, Variance, Skewness, and Kurtosis of ВGГM have given for $a=-2, b=3, u=1, \delta=1$, and $\lambda=2$ and various values of $\eta$ and $\beta$. From Table 3, we note that for fixed values of $a, b, u, \delta, \lambda$, and $\eta$, Mode 1 is decreasing, Median, Mode 2, and Mean are increasing functions of $\beta$. Also, for fixed values of $a, b, u, \delta, \beta$, and $\beta$, Mode 1 and Skewness are increasing and Mode 2 and Mean are decreasing functions of $\eta$.

3.3. Mean Residual Life and Mean Waiting Time. The mean residual life function, say $\varphi(t)$, is given by

$$
\begin{aligned}
\varphi(t)= & E(\tau-t \mid \tau>t) \\
= & \left(\frac{\operatorname{sign}(b-u)\left[\Gamma\left(\eta, \beta(|b-u| / \delta)^{\lambda}\right)-\Gamma\left(\eta, \beta(|u| / \delta)^{\lambda}\right)\right]-\operatorname{sign}(a-u)\left[\Gamma\left(\eta, \beta(|a-u| / \delta)^{\lambda}\right)-\Gamma\left(\eta, \beta(|u| / \delta)^{\lambda}\right)\right]}{\operatorname{sign}(b-u)\left[\Gamma\left(\eta, \beta(|b-u| / \delta)^{\lambda}\right)-\Gamma\left(\eta, \beta(|u| / \delta)^{\lambda}\right)\right]-\operatorname{sign}(t-u)\left[\Gamma\left(\eta, \beta(|t-u| / \delta)^{\lambda}\right)-\Gamma\left(\eta, \beta(|u| / \delta)^{\lambda}\right)\right]}\right. \\
& \times \frac{1}{2 \Gamma(\eta)(D(b)-D(a))}\left\{\frac{\delta}{\beta^{1 / \lambda}} \Gamma\left(\eta+\frac{1}{\lambda}\right)\left[\Gamma\left(\eta+\frac{1}{\lambda}, \beta\left(\frac{|b-u|}{\delta}\right)^{\lambda}\right)-\Gamma\left(\eta+\frac{1}{\lambda}, \beta\left(\frac{|t-u|}{\delta}\right)^{\lambda}\right)\right]\right. \\
& \left.\left. \pm u \Gamma(\eta)\left[\Gamma\left(\eta, \beta\left(\frac{|b-u|}{\delta}\right)^{\lambda}\right)-\Gamma\left(\eta, \beta\left(\frac{|t-u|}{\delta}\right)^{\lambda}\right)\right]\right\}\right)-t .
\end{aligned}
$$

The mean waiting time of $\mathrm{B} G \Gamma$ distribution, say $\bar{\varphi}(t)$, can be derived as

$$
\begin{aligned}
\bar{\varphi}(t)= & t-\frac{\int_{a}^{t} x \Upsilon(x \mid \Theta) \mathrm{d} x}{\Phi(t)} \\
= & t-\frac{1}{2 \Gamma(\eta)(D(b)-D(a))}\left\{\frac{\delta}{\beta^{1 / \lambda}} \Gamma\left(\eta+\frac{1}{\lambda}\right)\left[\Gamma\left(\eta+\frac{1}{\lambda}, \beta\left(\frac{|t-u|}{\delta}\right)^{\lambda}\right)-\Gamma\left(\eta+\frac{1}{\lambda}, \beta\left(\frac{|a-u|}{\delta}\right)^{\lambda}\right)\right]\right. \\
& \left. \pm u \Gamma(\eta)\left[\Gamma\left(\eta, \beta\left(\frac{|t-u|}{\delta}\right)^{\lambda}\right)-\Gamma\left(\eta, \beta\left(\frac{|a-u|}{\delta}\right)^{\lambda}\right)\right]\right\} \\
& \times \frac{\operatorname{sign}(b-u)\left[\Gamma\left(\eta, \beta(|b-u| / \delta)^{\lambda}\right)-\Gamma\left(\eta, \beta(|u| / \delta)^{\lambda}\right)\right]-\operatorname{sign}(a-u)\left[\Gamma\left(\eta, \beta(|a-u| / \delta)^{\lambda}\right)-\Gamma\left(\eta, \beta(|u| / \delta)^{\lambda}\right)\right]}{\operatorname{sign}(t-u)\left[\Gamma\left(\eta, \beta(|t-u| / \delta)^{\lambda}\right)-\Gamma\left(\eta, \beta(|u| / \delta)^{\lambda}\right)\right]-\operatorname{sign}(a-u)\left[\Gamma\left(\eta, \beta(|a-u| / \delta)^{\lambda}\right)-\Gamma\left(\eta, \beta(|u| / \delta)^{\lambda}\right)\right]} .
\end{aligned}
$$


TAble 2: Median, Mode, Mean, Variance, Skewness, and Kurtosis of BGГM.

\begin{tabular}{llccccccc}
\hline$\eta$ & $\lambda$ & Median & Mode1 & Mode2 & Mean & Variance & Skewness & Kurtosis \\
\hline 0.5 & 2 & 0.9979 & 1 & 1 & 0.9949 & 0.4895 & -0.0525 & 2.8857 \\
0.5 & 4 & 1.0037 & 1.7071 & 0.2929 & 1 & 0.5642 & 0 & 1.5708 \\
0.5 & 7 & 0.9909 & 1.8632 & 0.1368 & 1 & 0.6661 & 0 \\
2 & 2 & 0.4207 & 2.2248 & -0.2248 & 0.8932 & 1.8297 & 0.0117 & 1.2251 \\
2 & 4 & 0.8071 & 2.1502 & -0.1502 & 1 & 1.3293 & 0 & 1.13176489 \\
2 & 7 & 0.9244 & 2.0925 & -0.0924 & 1 & 1.1568 & 0 \\
7 & 2 & -1.3817 & 3.5495 & -1.5495 & -0.9009 & 2.0185 & 2.0649 \\
7 & 4 & -0.1453 & 2.6119 & -0.6119 & 0.9959 & 2.5958 & 0.0035 \\
7 & 7 & 0.9785 & 2.3166 & -0.3166 & 1 & 1.7181 & 04581787 \\
\hline
\end{tabular}

TAble 3: Median, Mode, Mean, Variance, Skewness, and Kurtosis of BGГM.

\begin{tabular}{lcccccccc}
\hline$\eta$ & $\beta$ & Median & Mode1 & Mode2 & Mean & Variance & Skewness & Kurtosis \\
\hline 0.5 & 0.5 & 0.9732 & 1 & 1 & 0.9492 & 0.8732 & -0.1824 \\
0.5 & 2 & 1 & 1 & 1 & 0.9999 & 0.2499 & -0.002 \\
0.5 & 7 & 1 & 1 & 1 & 1 & 0.0714 & 0 \\
2 & 0.5 & -0.2227 & 2.7321 & -0.7321 & 0.4599 & 2.7085 & 0.29472 \\
2 & 2 & 0.8327 & 1.866 & 0.134 & 0.9968 & 0.9946 & -0.0052 & 1.4377 \\
2 & 7 & 1.001 & 1.4629 & 0.5371 & 1 & 0.2857 & 0 \\
7 & 0.5 & -1.722 & 4.6056 & -2.6056 & -1.547 & 0.5886 & 4.8737 \\
7 & 2 & -0.483 & 2.8028 & -0.8028 & 0.5854 & 3.0344 & 0.3259 \\
7 & 7 & 1.0855 & 1.9636 & 0.0364 & 1 & 1 & 28.0326 \\
\hline
\end{tabular}

3.4. Entropy. The entropy of a random variable $X$ measures the variation of the uncertainty. The Rényi entropy of BGГ distribution, say $\operatorname{RE}_{X}(\nu)$ for $\nu \neq 1$ and $\nu>0$, is derived as

$$
\begin{aligned}
\operatorname{RE}_{X}(\nu)= & \frac{\ln \int_{a}^{b} x \Upsilon^{\nu}(x \mid \Theta) \mathrm{d} x}{1-\nu} \\
= & -\ln \lambda-\frac{\nu \ln 2}{1-\nu}+\ln \delta-\frac{\nu \ln \Gamma(\eta)}{1-\nu} \\
& -\frac{v \ln (D(b)-D(a))}{1-\nu}-\frac{\ln \beta}{\lambda}-\left(\frac{1}{\lambda}+\frac{\nu \eta}{1-\nu}\right) \ln v \\
& +\frac{1}{1-\nu} \ln \Gamma\left(\nu \eta-\frac{\nu-1}{\lambda}\right)+\frac{1}{1-\nu} \ln \left\{ \pm \Gamma\left(\nu \eta-\frac{v-1}{\lambda}, \beta \nu\left(\frac{|b-u|}{\delta}\right)^{\lambda}\right) \mp \Gamma\left(\nu \eta-\frac{v-1}{\lambda}, \beta \nu\left(\frac{|a-u|}{\delta}\right)^{\lambda}\right)\right\} .
\end{aligned}
$$

3.5. Order Statistics. Let $X_{1: n}, X_{2: n}, \ldots, X_{n: n}$ denote the from BGГ distribution. The probability density function of order statistics obtained from a random sample of size $n \quad i^{\text {th }}$ order statistics is given by

$$
\begin{aligned}
f_{i: n}(x) & =\frac{n !}{(i-1) !(n-i) !}(\Phi(x))^{i-1}(1-\Phi(x))^{n-i} \Upsilon(x \mid \Theta) \\
& =\frac{n ! \lambda \beta^{\eta}(|x-u| / \delta)^{\eta \lambda-1} e^{-\beta(|x-u| / \delta)^{\lambda}}(D(x)-D(a))^{i-1}(D(b)-D(x))^{n-i}}{2(i-1) !(n-i) ! \delta \Gamma(\eta)(D(b)-D(a))^{n}} .
\end{aligned}
$$


The pdf of the minimum and the maximum order statistics of ВGГ distribution can be obtained, respectively, as follows:

$$
\begin{aligned}
& f_{1: n}(x)=\frac{n \lambda \beta^{\eta}(|x-u| / \delta)^{\eta \lambda-1} e^{-\beta(|x-u| / \delta)^{\lambda}}(D(b)-D(x))^{n-1}}{2 \delta \Gamma(\eta)(D(b)-D(a))^{n}}, \\
& f_{n: n}(x)=\frac{n \lambda \beta^{\eta}(|x-u| / \delta)^{\eta \lambda-1} e^{-\beta(|x-u| / \delta)^{\lambda}}(D(x)-D(a))^{n-1}}{2 \delta \Gamma(\eta)(D(b)-D(a))^{n}} .
\end{aligned}
$$

If $n$ is odd. The pdf of ВGГ distribution of the median is obtained by substituting $i=(n+1) / 2$ in equation (24) as follows:

$$
f_{(n+1 / 2): n}(x)=\frac{n ! \lambda \beta^{\eta}(|x-u| / \delta)^{\eta \lambda-1} e^{-\beta(|x-u| / \delta)^{\lambda}}(D(x)-D(a))^{n-1 / 2}(D(b)-D(x))^{n-1 / 2}}{2((n-1 / 2) !)^{2} \delta \Gamma(\eta)(D(b)-D(a))^{n}} .
$$

The joint pdf of the $i^{t h}$ and the $l^{\text {th }}$ order statistics for $x<y$ can be written as

$$
\begin{aligned}
f_{i, l: n}(x)= & \frac{n !}{(i-1) !(l-i-1) !(n-l) !}(\Phi(x))^{i-1} \\
& (1-\Phi(y))^{n-l}(\Phi(y)-\Phi(x))^{l-i-1} \Upsilon(x \mid \Theta) \Upsilon(y \mid \Theta)
\end{aligned}
$$

So the joint pdf of the $i^{\text {th }}$ and the $l^{\text {th }}$ order statistics of BGГ distribution is

$$
\begin{aligned}
f_{i, l: n}(x)= & \frac{n ! \lambda^{2} \beta^{2 \eta}(D(x)-D(a))^{i-1}(D(b)-D(y))^{n-l}(D(y)-D(x))^{l-i-1}}{4 \delta^{2} \Gamma^{2}(\eta)(D(b)-D(a))^{n}(i-1) !(l-i-1) !(n-l) !} \\
& \times\left(\frac{|x-u \| y-u|}{\delta^{2}}\right)^{\eta \lambda-1} e^{-\beta\left((|x-u| \mid \delta)^{\lambda}+(|y-u| \mid \delta)^{\lambda}\right)} .
\end{aligned}
$$

\section{Maximizing the Log-Likelihood Function}

Here, we consider the estimation of the unknown parameters of the ВGГD by the method of maximum likelihood. Let $x_{1}, x_{2}, \ldots, x_{N}$ be a random sample from the BGГD. The total log-likelihood $(L(\Theta))$ is given by

$$
L(\Theta)=\sum_{i=1}^{N}\left[\ln T\left(x_{i} \mid u, \delta, \beta, \eta, \lambda\right)-\ln \int_{\Omega} T(x \mid u, \delta, \beta, \eta, \lambda) \mathrm{d} x\right] .
$$

4.1. Location Parameter Estimation. To maximize the likelihood function in (28), we consider the derivation of $L$ with the location $u$ at the $(t+1)$ iteration step. We have

$$
\begin{aligned}
\frac{\partial L}{\partial u}= & \sum_{i=1}^{N}\left\{\left(\left(u-x_{i}\right)\left|u-x_{i}\right|^{-2}\left(\eta \lambda-1-\frac{\beta \lambda}{\delta^{\lambda}}\left(\left|u-x_{i}\right|\right)^{\lambda}\right)\right)\right. \\
& \left.-\frac{\int_{\Omega}\left(\operatorname{sign}\left(u-x_{i}\right)\left|u-x_{i}\right|^{-1}\left(\eta \lambda-1-\beta \lambda / \delta^{\lambda}\left(\left|u-x_{i}\right|\right)^{\lambda}\right)\right) T(x \mid \Theta) \mathrm{d} x}{\int_{\Omega} T(x \mid \Theta) \mathrm{d} x}\right\}
\end{aligned}
$$


At that point as [23], we have

$$
\int_{\Omega} T(x \mid \Theta) \mathrm{d} x \approx \frac{1}{M} \sum_{i=1}^{M} 1_{\Omega}\left(v_{i}\right),
$$

where $v_{i} \sim T\left(x \mid \Theta^{(t)}\right)$ indicates the random variable that is drawn from the probability distribution $T\left(x \mid \Theta^{(t)}\right)$, with $\Theta^{(t)}=\left(u^{(t)}, \delta^{(t)}, \beta^{(t)}, \eta^{(t)}, \lambda^{(t)}\right) \prime$ and $M$ is the number of random variables $v_{i}$. We use $M=10^{6}$, for our experiments. In the same manner, we can write

$$
\begin{aligned}
& \int_{\Omega}\left(\operatorname{sign}\left(u-x_{i}\right)\left|u-x_{i}\right|^{-1}\left(\eta \lambda-1-\frac{\beta \lambda}{\delta^{\lambda}}\left(\left|u-x_{i}\right|\right)^{\lambda}\right)\right) T(x \mid \Theta) \mathrm{d} x \\
& \quad \approx \frac{1}{M} \sum_{i=1}^{M} 1_{\Omega}\left(v_{i}\right)\left(\operatorname{sign}\left(u^{(t)}-v_{i}\right)\left|u^{(t)}-v_{i}\right|^{-1}\left(\eta^{(t)} \lambda^{(t)}-1-\frac{\beta^{(t)} \lambda^{(t)}}{\left(\delta^{(t)}\right)^{\lambda^{(t)}}}\left(\left|u^{(t)}-v_{i}\right|\right)^{\lambda^{(t)}}\right)\right) .
\end{aligned}
$$

By using (31) and (32), we can rewrite (30) as

$$
\left.\frac{\partial L}{\partial u^{\prime}}\right|_{u=u^{(t)}} \approx \sum_{i=1}^{N}\left|u^{(t)}-x_{i}\right|^{-2}\left\{\left(\left(u^{(t)}-x_{i}\right)\left(\eta^{(t)} \lambda^{(t)}-1-\frac{\beta^{(t)} \lambda^{(t)}}{\left(\delta^{(t)}\right)^{\lambda^{(t)}}}\left(\left|u^{(t)}-x_{i}\right|\right)^{\lambda^{(t)}}\right)\right)-H\left|u^{(t)}-x_{i}\right|^{2}\right\},
$$

where

$$
\begin{aligned}
& H=\frac{\sum_{i=1}^{M} 1_{\Omega}\left(v_{i}\right) \operatorname{sign}\left(u^{(t)}-v_{i}\right)\left|u^{(t)}-v_{i}\right|^{-1}\left(\eta^{(t)} \lambda^{(t)}-1-\beta^{(t)} \lambda^{(t)} /\left(\delta^{(t)}\right)^{\lambda^{(t)}}\left(\left|u^{(t)}-v_{i}\right|\right)^{\lambda^{(t)}}\right)}{\sum_{i=1}^{M} 1_{\Omega}\left(v_{i}\right)} . \\
& \text { the theory of robust statistics [24], any } \quad u=\frac{\sum_{i} \omega_{i} x_{i}}{\sum_{i} \omega_{i}} ; \text { where } \omega_{i}=\frac{F\left(x_{i}-u\right)}{x_{i}-u} .
\end{aligned}
$$

According to the theory of robust statistics [24], any estimate $u$ is defined by an implicit equation:

$$
\sum_{i} F\left(x_{i}-u\right)=0 .
$$

Now, we can apply (35) to $\partial L / \partial u$ in (33), and the solution of $\partial L / \partial u=0$ gives the solutions of $u$ at the $(t+1)$ step:

This gives a numerical solution of the location of $u$ as a weighted mean:

$$
u^{(t+1)}=\frac{\sum_{i=1}^{N}\left[\left|u^{(t)}-x_{i}\right|^{-2} x_{i}\left(\eta^{(t)} \lambda^{(t)}-1-\left(\beta^{(t)} \lambda^{(t)} /\left(\delta^{(t)}\right)^{\lambda^{(t)}}\right)\left(\left|u^{(t)}-x_{i}\right|\right)^{\lambda^{(t)}}\right)+H\right]}{\sum_{i=1}^{N}\left[\left|u^{(t)}-x_{i}\right|^{-2}\left(\eta^{(t)} \lambda^{(t)}-1-\left(\beta^{(t)} \lambda^{(t)} /\left(\delta^{(t)}\right)^{\lambda^{(t)}}\right)\left(\left|u^{(t)}-x_{i}\right|\right)^{\lambda^{(t)}}\right)\right]} .
$$


4.2. Scale Parameters Estimation. Putting the derivative of the log-likelihood function $L$ with respect to the scale parameter $\delta$ at the $(t+1)$ iteration step, we have

$$
\begin{aligned}
& \frac{\partial L}{\partial \delta}= \delta^{-1} \sum_{i=1}^{N}\left[\beta \lambda \delta^{-\lambda}\left|u-x_{i}\right|^{\lambda}-\eta \lambda\right. \\
&\left.-\frac{\int_{\Omega}\left(\beta \lambda \delta^{-\lambda}\left|u-x_{i}\right|^{\lambda}-\eta \lambda\right) T(x \mid \Theta) \mathrm{d} x}{\int_{\Omega} T(x \mid \Theta) \mathrm{d} x}\right] .
\end{aligned}
$$

Similarly as (31) and (32), we can rewrite $\partial L / \partial \delta$ as

$$
\frac{\partial L}{\partial \delta}=\delta^{-1} \sum_{i=1}^{N}\left[\beta \lambda \delta^{-\lambda}\left|u-x_{i}\right|^{\lambda}-\eta \lambda-G\right]
$$

where

$$
G=\frac{\sum_{i=1}^{M}\left[\beta^{(t)} \lambda^{(t)}\left(\delta^{(t)}\right)^{-\lambda^{(t)}}\left|u^{(t)}-v_{i}\right|^{\lambda^{(t)}}-\eta^{(t)} \lambda^{(t)}\right] 1_{\Omega}\left(v_{i}\right)}{\sum_{i=1}^{M} 1_{\Omega}\left(v_{i}\right)}
$$

The solution of $\partial L / \partial \delta=0$ yields the solutions of $\delta$ at the $(t+1)$ step:

$$
\delta^{(t+1)}=\left(\frac{\beta^{(t)} \lambda^{(t)} \sum_{i=1}^{N}\left|u^{(t)}-x_{i}\right|^{\lambda^{(t)}}}{\sum_{i=1}^{N}\left(\eta^{(t)} \lambda^{(t)}+G\right)}\right)^{1 / \lambda^{(t)}} .
$$

The next step is to update the estimate of the scale parameter $\beta$. This includes fixing the other parameters and improving the estimate of $\beta$ by using the Newton Raphson method [25]. Every cycle requires the first and second derivatives of $L(\Theta)$ with respect to the parameter $\beta$.

$$
\beta^{(t+1)}=\beta^{(t)}-\left.\frac{\partial L / \partial \beta}{\left(\partial^{2} L / \partial \beta^{2}\right)+\varepsilon}\right|_{\beta=\beta^{(t)}}
$$

where $\varepsilon$ is a scaling element. The derivative of the function $L(\Theta)$ regarding $\beta$ is given by

$$
\frac{\partial L}{\partial \beta}=\sum_{i=1}^{N}\left\{f\left(x_{i}, \Theta\right)-\frac{\int_{\Omega} f(x, \Theta) T(x \mid \Theta) \mathrm{d} x}{\int_{\Omega} T(x \mid \Theta) \mathrm{d} x}\right\},
$$

where

$$
f\left(x_{i}, \Theta\right)=\frac{1}{T\left(x_{i} \mid \Theta\right)} \frac{\partial T\left(x_{i} \mid \Theta\right)}{\partial \beta}=\frac{\eta}{\beta}-\left(\frac{\left|u-x_{i}\right|}{\delta}\right)^{\lambda} .
$$

The term $\partial L / \partial \beta$ can be approximated as

$$
\left.\frac{\partial L}{\partial \beta}\right|_{\beta=\beta^{(t)}} \approx \sum_{i=1}^{N}\left\{f\left(x_{i}, \Theta^{(t)}\right)-\frac{\sum_{i=1}^{M} 1_{\Omega}\left(v_{i}\right) f\left(v_{i}, \Theta^{(t)}\right)}{\sum_{i=1}^{M} 1_{\Omega}\left(v_{i}\right)}\right\} \text {. }
$$

The term $\partial^{2} L / \partial \beta^{2}$ is given by

$$
\begin{aligned}
\frac{\partial^{2} L}{\partial \beta^{2}}= & \sum_{i=1}^{N}\left\{\frac{\partial f(x, \Theta)}{\partial \beta}+\frac{\left(\int_{\Omega} T(x \mid \Theta) f \mathrm{~d} x\right)^{2}}{\left(\int_{\Omega} T(x \mid \Theta) \mathrm{d} x\right)^{2}}\right. \\
& \left.-\frac{\int_{\Omega}\left((\partial f / \partial \beta)+f^{2}\right) T(x \mid \Theta) \mathrm{d} x}{\int_{\Omega} T(x \mid \Theta) \mathrm{d} x}\right\},
\end{aligned}
$$

where

$$
\frac{\partial f}{\partial \beta}=\frac{-\eta}{\beta^{2}}
$$

Also the term $\partial^{2} L / \partial \beta^{2}$ can be approximated as

$$
\begin{aligned}
\left.\frac{\partial^{2} L}{\partial \beta^{2}}\right|_{\beta=\beta^{(t)}} \approx & \sum_{i=1}^{N}\left\{\frac{-\eta^{(t)}}{\left(\beta^{(t)}\right)^{2}}+\frac{\left(\sum_{i=1}^{M} 1_{\Omega}\left(v_{i}\right)\left(f\left(v_{i}, \Theta^{(t)}\right)\right)\right)^{2}}{\left(\sum_{i=1}^{M} 1_{\Omega}\left(v_{i}\right)\right)^{2}}\right. \\
& \left.-\frac{\sum_{i=1}^{M} 1_{\Omega}\left(v_{i}\right)\left(-\eta^{(t)} /\left(\beta^{(t)}\right)^{2}+\left(f\left(v_{i}, \Theta^{(t)}\right)\right)^{2}\right)}{\sum_{i=1}^{M} 1_{\Omega}\left(v_{i}\right)}\right\} .
\end{aligned}
$$

4.3. Shape Parameters Estimation. For shape parameter estimation $\eta$ by using the Newton Raphson method, we have

$$
\eta^{(t+1)}=\eta^{(t)}-\left.\frac{\partial L / \partial \eta}{\left(\partial^{2} L / \partial \eta^{2}\right)+\varepsilon}\right|_{\eta=\eta^{(t)}}
$$

The derivative of the function $L(\Theta)$ with respect to $\eta$ is given by

$$
\frac{\partial L}{\partial \eta}=\sum_{i=1}^{N}\left\{g\left(x_{i} \mid \Theta\right)-\frac{\int_{\Omega} g(x \mid \Theta) T(x \mid \Theta) \mathrm{d} x}{\int_{\Omega} T(x \mid \Theta) \mathrm{d} x}\right\},
$$

where

$$
\begin{aligned}
g\left(x_{i} \mid \Theta\right) & =\frac{1}{T\left(x_{i} \mid \Theta\right)} \frac{\partial T\left(x_{i} \mid \Theta\right)}{\partial \eta} \\
& =\ln \beta-\Psi(\eta)+\lambda\left(\ln \left|u-x_{i}\right|-\ln \delta\right) .
\end{aligned}
$$

The term $\partial L / \partial \eta$ can be approximated as

$$
\left.\frac{\partial L}{\partial \eta}\right|_{\eta=\eta^{(t)}} \approx \sum_{i=1}^{N}\left\{g^{(t)}\left(x_{i} \mid \Theta\right)-\frac{\sum_{m=1}^{M} 1_{\Omega}\left(v_{m}\right) g^{(t)}\left(v_{m} \mid \Theta\right)}{\sum_{i=1}^{M} 1_{\Omega}\left(v_{i}\right)}\right\},
$$

where

$$
g^{(t)}\left(x_{i} \mid \Theta\right)=\ln \beta^{(t)}-\Psi\left(\eta^{(t)}\right)+\lambda^{(t)}\left(\ln \left|u^{(t)}-x_{i}\right|-\ln \delta^{(t)}\right) .
$$

The calculation of the term $\partial^{2} L / \partial \eta^{2}$ is obtained as 


$$
\begin{aligned}
\frac{\partial^{2} L}{\partial \eta^{2}}= & \sum_{i=1}^{N}\left\{\frac{\partial g\left(x_{i} \mid \Theta\right)}{\partial \eta}+\frac{\left(\int_{\Omega} T(x \mid \Theta) g(x \mid \Theta) \mathrm{d} x\right)^{2}}{\left(\int_{\Omega} T(x \mid \Theta) \mathrm{d} x\right)^{2}}\right. \\
& \left.-\frac{\int_{\Omega}\left(\partial g(x \mid \Theta) / \partial \eta+g^{2}(x \mid \theta)\right) T(x \mid \Theta) \mathrm{d} x}{\int_{\Omega} T(x \mid \Theta) \mathrm{d} x}\right\},
\end{aligned}
$$

where

$$
\frac{\partial g}{\partial \eta}=-\Psi \prime(\eta)
$$

The term $\partial^{2} L / \partial \eta^{2}$ can be approximated as

$$
\begin{aligned}
\left.\frac{\partial^{2} L}{\partial \eta^{2}}\right|_{\eta=\eta^{(t)}} \approx & \sum_{i=1}^{N}\left\{\left(\frac{\partial g\left(x_{i} \mid \Theta\right)}{\partial \eta}\right)^{(t)}+\frac{\left(\sum_{m=1}^{M} 1_{\Omega}\left(v_{m}\right) g^{(t)}\left(v_{m} \mid \Theta\right)\right)^{2}}{\left(\sum_{i=1}^{M} 1_{\Omega}\left(v_{i}\right)\right)^{2}}\right. \\
& \left.-\frac{\sum_{m=1}^{M} 1_{\Omega}\left(v_{m}\right)\left(\left(\partial g\left(v_{m} \mid \Theta\right) / \partial \eta\right)^{(t)}+\left(g^{(t)}\left(v_{m} \mid \Theta\right)\right)^{2}\right)}{\sum_{i=1}^{M} 1_{\Omega}\left(v_{i}\right)}\right\},
\end{aligned}
$$

where

$$
\left(\frac{\partial g\left(x_{i} \mid \theta\right)}{\partial \eta}\right)^{(t)}=-\Psi^{\prime}\left(\eta^{(t)}\right)
$$

For shape parameter estimation $\lambda$ by using the Newton Raphson method, we have

$$
\lambda^{(t+1)}=\lambda^{(t)}-\left.\frac{\partial L / \partial \lambda}{\partial^{2} L / \partial \lambda^{2}+\varepsilon}\right|_{\lambda=\lambda^{(t)}} .
$$

The derivative of the function $L(\Theta)$ with respect to $\lambda$ is given by

$$
\frac{\partial L}{\partial \lambda}=\sum_{i=1}^{N}\left\{h\left(x_{i} \mid \Theta\right)-\frac{\int_{\Omega} h(x \mid \Theta) T(x \mid \Theta) \mathrm{d} x}{\int_{\Omega} T(x \mid \Theta) \mathrm{d} x}\right\},
$$

where

$$
\begin{aligned}
h\left(x_{i} \mid \Theta\right) & =\frac{1}{T\left(x_{i} \mid \Theta\right)} \frac{\partial T\left(x_{i} \mid \Theta\right)}{\partial \lambda} \\
& =\frac{1}{\lambda}+\left(\eta-\beta\left(\frac{\left|u-x_{i}\right|}{\delta}\right)^{\lambda}\right) \ln \left(\frac{\left|u-x_{i}\right|}{\delta}\right) .
\end{aligned}
$$

The term $\partial L / \partial \lambda$ can be approximated as

$$
\left.\frac{\partial L}{\partial \lambda}\right|_{\lambda=\lambda^{(t)}} \approx \sum_{i=1}^{N}\left\{h^{(t)}\left(x_{i} \mid \Theta\right)-\frac{\sum_{m=1}^{M} 1_{\Omega}\left(v_{m}\right) h^{(t)}\left(v_{m} \mid \Theta\right)}{\sum_{m=1}^{M} 1_{\Omega}\left(v_{m}\right)}\right\},
$$

where

$$
h^{(t)}\left(x_{i} \mid \Theta\right)=\frac{1}{\lambda^{(t)}}+\left(\eta^{(t)}-\beta^{(t)}\left(\frac{\left|u^{(t)}-x_{i}\right|}{\delta^{(t)}}\right)^{\lambda^{(t)}}\right) \ln \left(\frac{\left|u^{(t)}-x_{i}\right|}{\delta^{(t)}}\right) .
$$

The calculation of the term $\partial^{2} L / \partial \lambda^{2}$ is obtained as

$$
\begin{aligned}
\frac{\partial^{2} L}{\partial \lambda^{2}}= & \sum_{i=1}^{N}\left\{\frac{\partial h\left(x_{i} \mid \Theta\right)}{\partial \lambda}+\frac{\left(\int_{\Omega} T(x \mid \Theta) h(x \mid \Theta) \mathrm{d} x\right)^{2}}{\left(\int_{\Omega} T(x \mid \Theta) \mathrm{d} x\right)^{2}} N\right. \\
& \left.-\frac{\int_{\Omega}\left((\partial h(x \mid \Theta) / \partial \lambda)+h^{2}(x \mid \Theta)\right) T(x \mid \Theta) \mathrm{d} x}{\int_{\Omega} T(x \mid \Theta) \mathrm{d} x}\right\},
\end{aligned}
$$

where

$$
\frac{\partial h}{\partial \lambda}=\frac{-1}{\lambda^{2}}-\beta\left(\frac{\left|u-x_{i}\right|}{\delta}\right)^{\lambda} \ln ^{2}\left(\frac{\left|u-x_{i}\right|}{\delta}\right) .
$$

The term $\partial^{2} L / \partial \lambda^{2}$ can be approximated as

$$
\begin{aligned}
\left.\frac{\partial^{2} L}{\partial \lambda^{2}}\right|_{\lambda=\lambda^{(t)}} \approx & \sum_{i=1}^{N}\left\{\left(\frac{\partial h\left(x_{i} \mid \Theta\right)}{\partial \lambda}\right)^{(t)}+\frac{\left(\sum_{m=1}^{M} 1_{\Omega}\left(v_{m}\right) h^{(t)}\left(v_{m} \mid \Theta\right)\right)^{2}}{\left(\sum_{m=1}^{M} 1_{\Omega}\left(v_{m}\right)\right)^{2}}\right. \\
& \left.\frac{\sum_{m=1}^{M} 1_{\Omega}\left(v_{m}\right)\left(\left(\partial h\left(v_{m} \mid \Theta\right) / \partial \lambda\right)^{(t)}+\left(h^{(t)}\left(v_{m} \mid \Theta\right)\right)^{2}\right)}{\sum_{m=1}^{M} 1_{\Omega}\left(v_{m}\right)}\right\} .
\end{aligned}
$$

4.4. Algorithm. To study the stability of our model, we have to find the set of initial points that generate a convergent sequence which called stable points of the dynamical system, i.e., we have to find $u^{(0)}, \delta^{(0)}, \beta^{(0)}, \eta^{(0)}, \lambda^{(0)}$ such that $\lim _{t \rightarrow \infty} \mathcal{u}^{(t)}, \lim _{t \rightarrow \infty} \delta^{(t)}, \lim _{t \rightarrow \infty} \beta^{(t)}, \lim _{t \rightarrow \infty} \eta^{(t)}$, and $\lim _{t \rightarrow \infty} \lambda^{(t)}$ exist. Indeed for fixed initial, it is difficult to predict how the approximation sequence behaves; hence, for this purpose, we take a random numbers of initial points until the convergence is verified (two successive approximations of each parameter correct to 4 decimal places). The various steps of the proposed model can be summarized as follows:

Step 1: Initialize the parameters $\Theta(u, \delta, \beta, \eta, \lambda)$.

Step 2: Reestimate the parameters $\Theta(u, \delta, \beta, \eta, \lambda)$, where the most common value of scaling parameter $\varepsilon$ is $10^{-30}$ for our experiments.

+Update the parameter $u$ in (37). + Update the parameter $\delta$ in (41). + Update the parameter $\beta$ in (42). + Update the parameter $\eta$ in (49). + Update the parameter $\lambda$ in (58). 


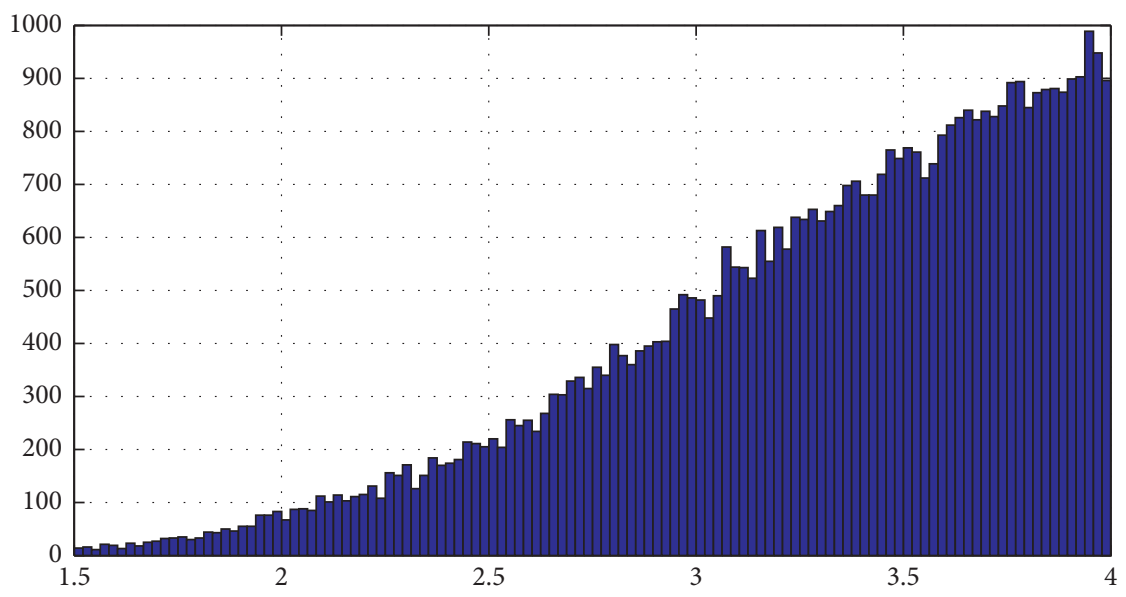

(a)

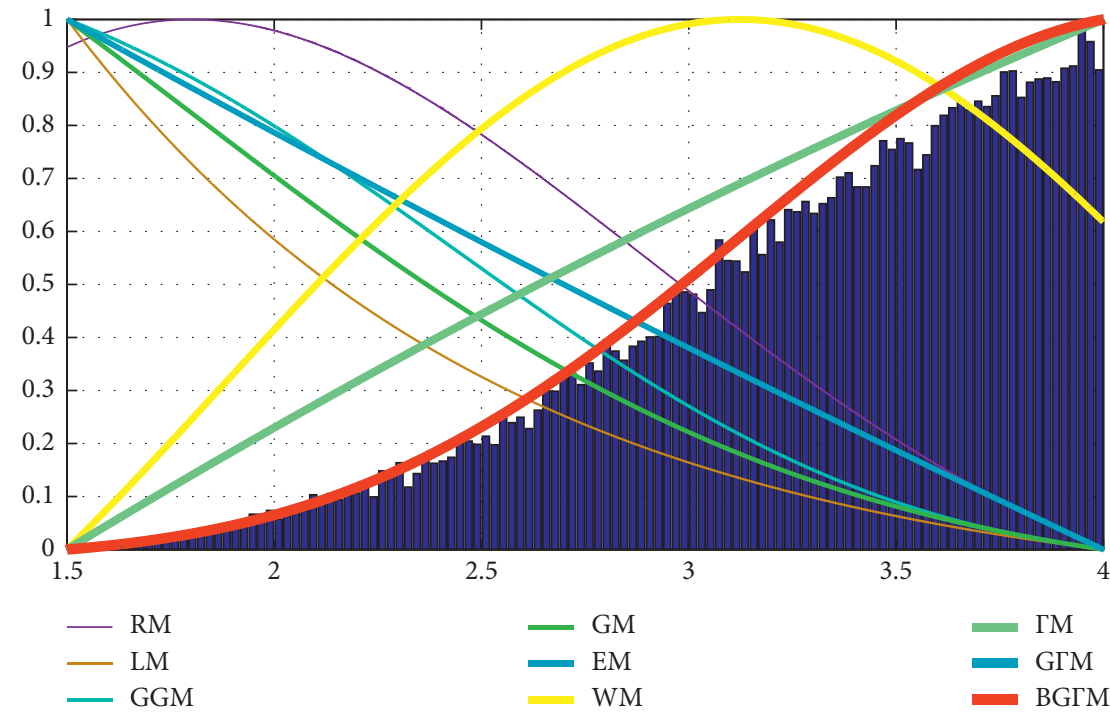

(b)

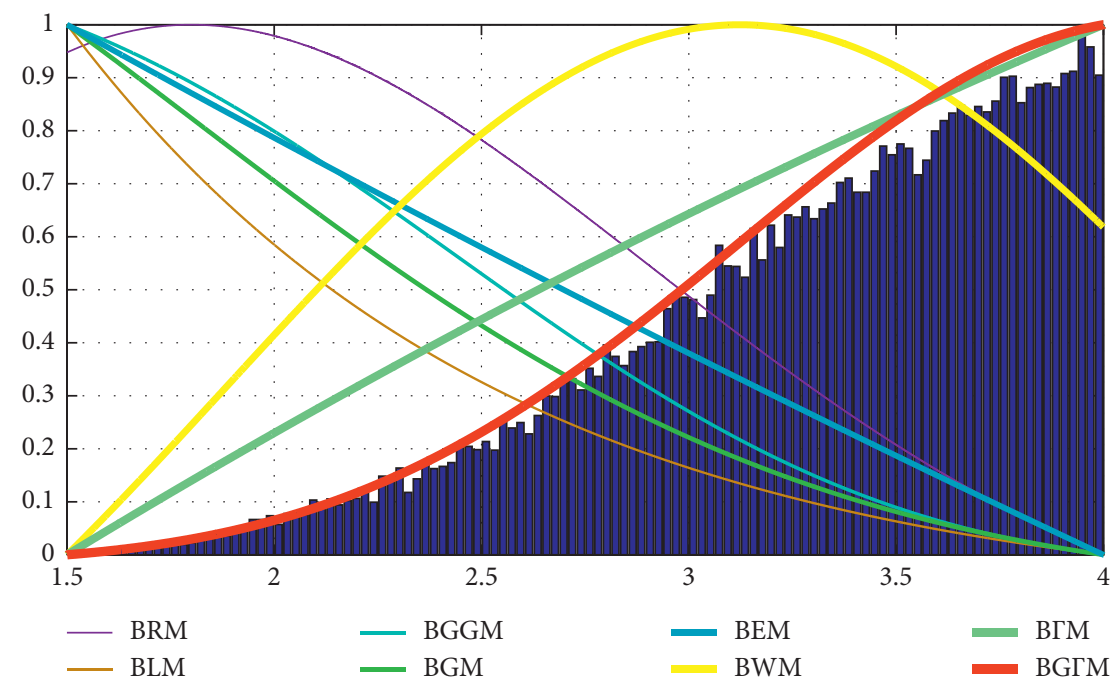

(c)

FIgURE 4: The estimated histogram (a) the histogram of the observed data with $u=0.3 ; \delta=1.5 ; \eta=2, \lambda=3$ and $\beta=0.1$ in the interval $(1.5,4)$; (b) the estimated histogram of RM, LM, WM, GM, EM, GGM, ГM, GГM, and GГM; (c) the estimated histogram of BRM, BLM, BWM, BGM, BEM, BGGM, BГM, and BGГM. 


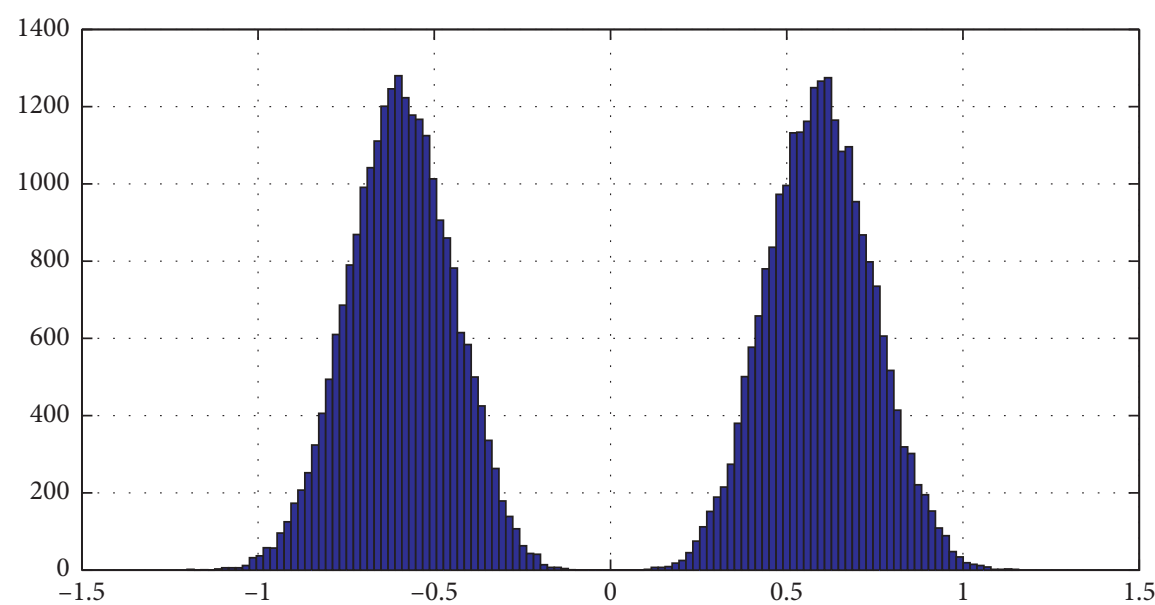

(a)

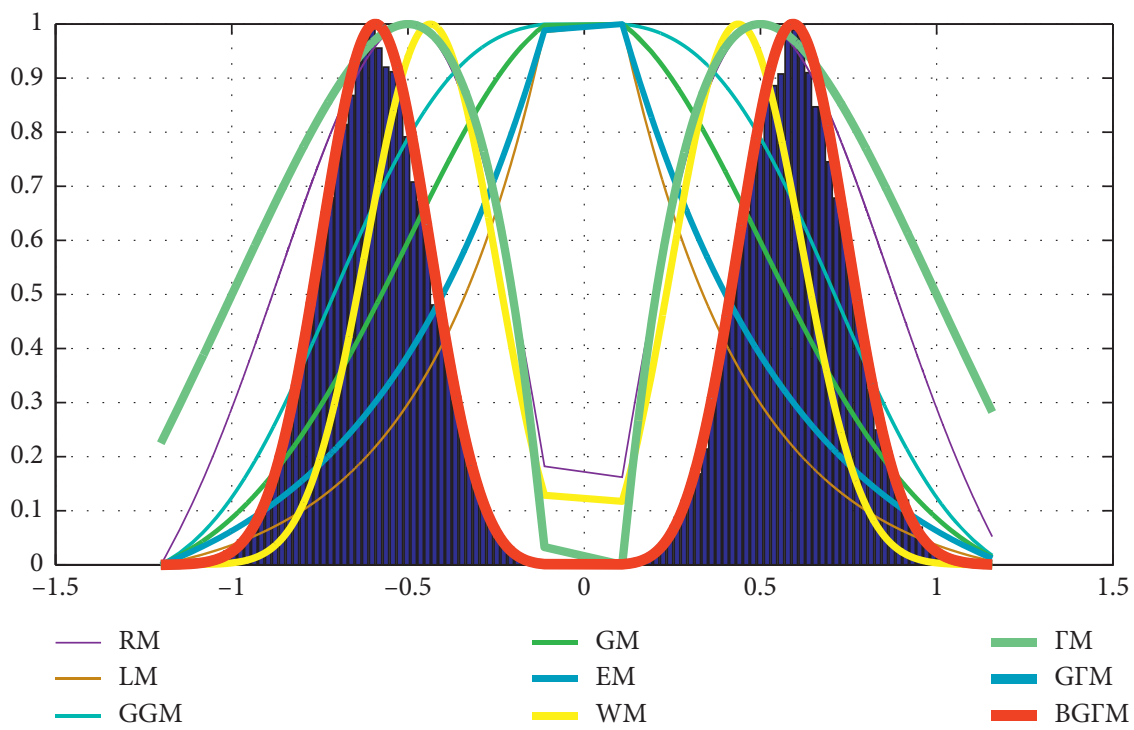

(b)

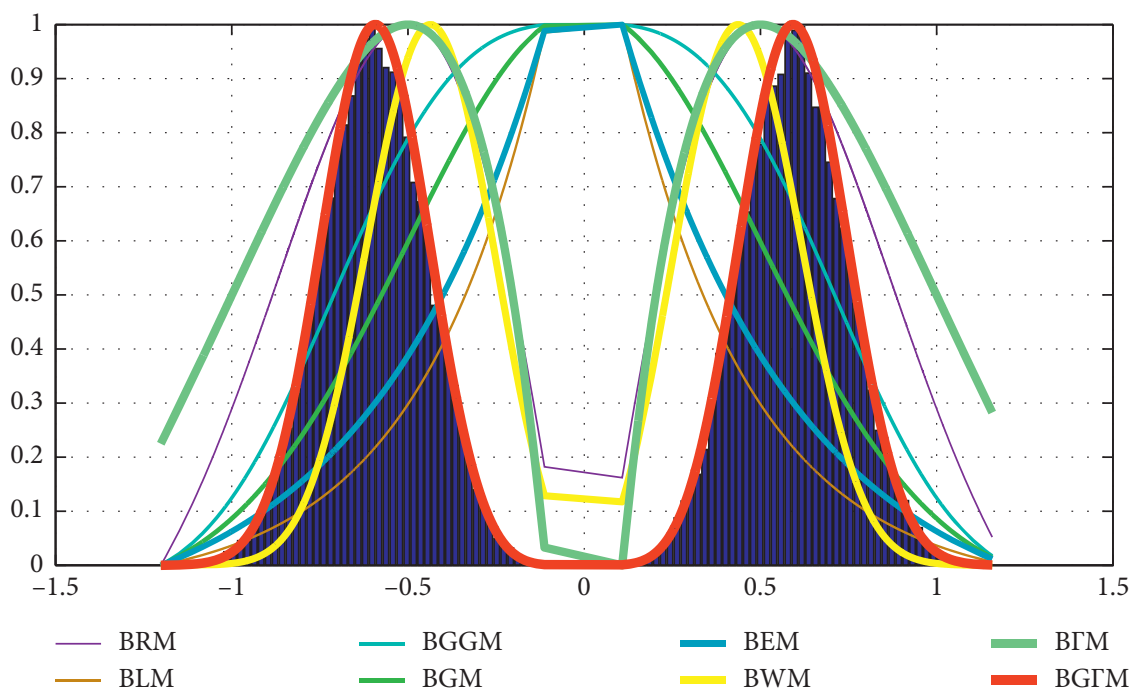

(c)

Figure 5: The estimated histogram. (a) The histogram of the observed data with $u=0 ; \delta=0.5 ; \eta=2, \lambda=3$ and $\beta=1$ in the interval $(-1.5,1.5)$; (b) the estimated histogram of RM, LM, WM, GM, EM, GGM, ГM, GГM, and GГM; (c) the estimated histogram of BRM, BLM, BWM, BGM, BEM, BGGM, BГM, and BGГM. 


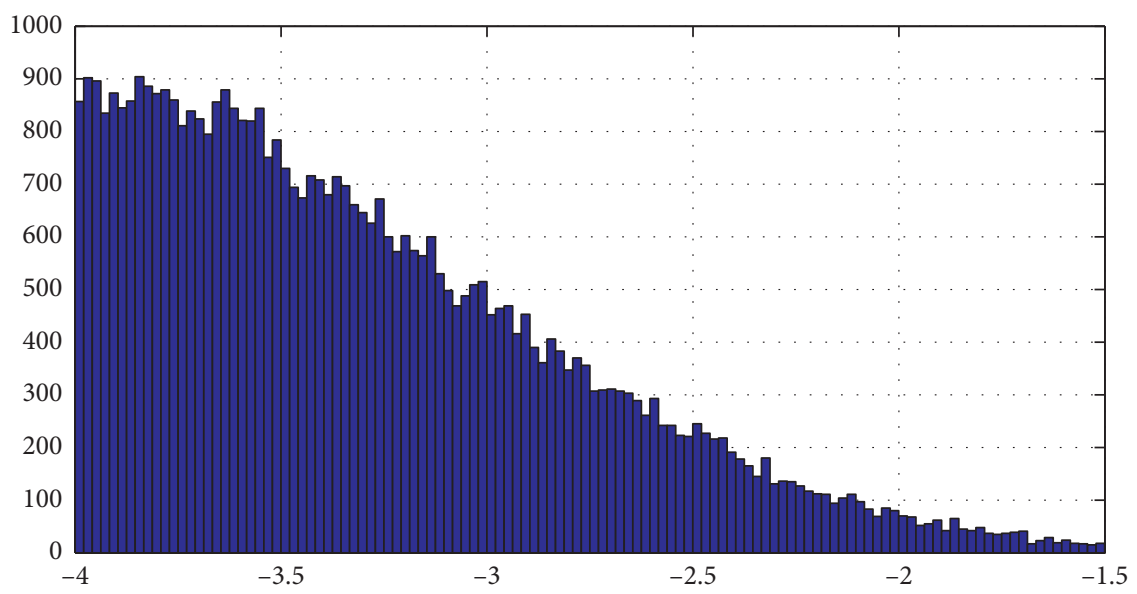

(a)

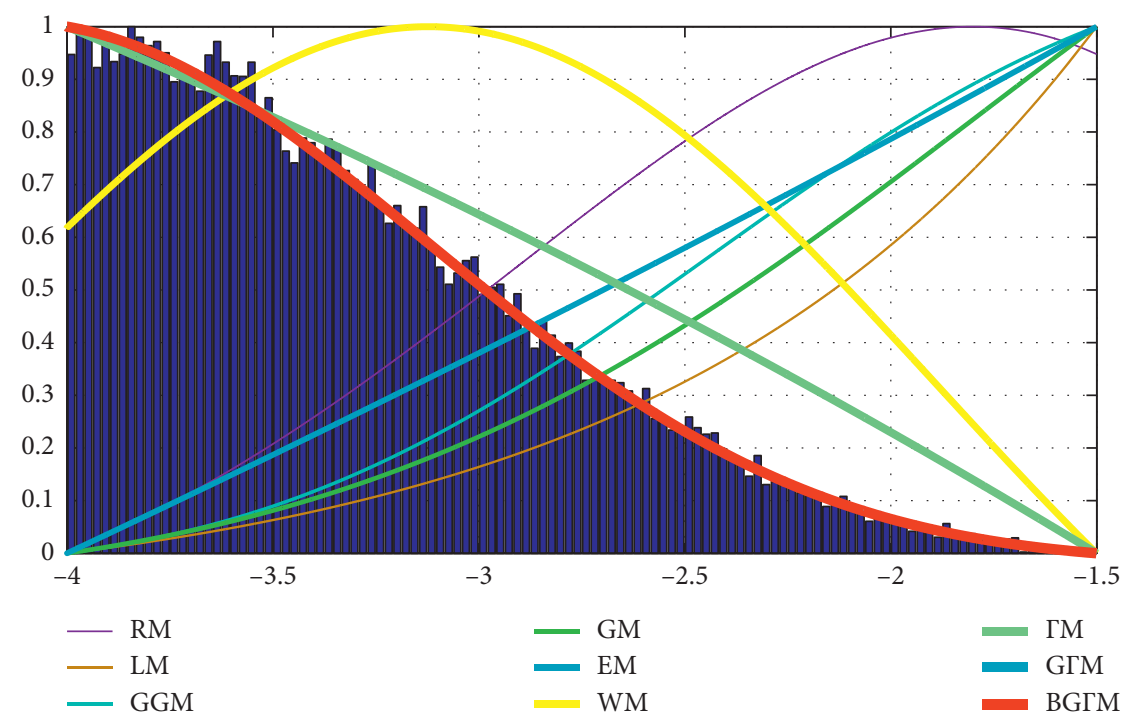

(b)

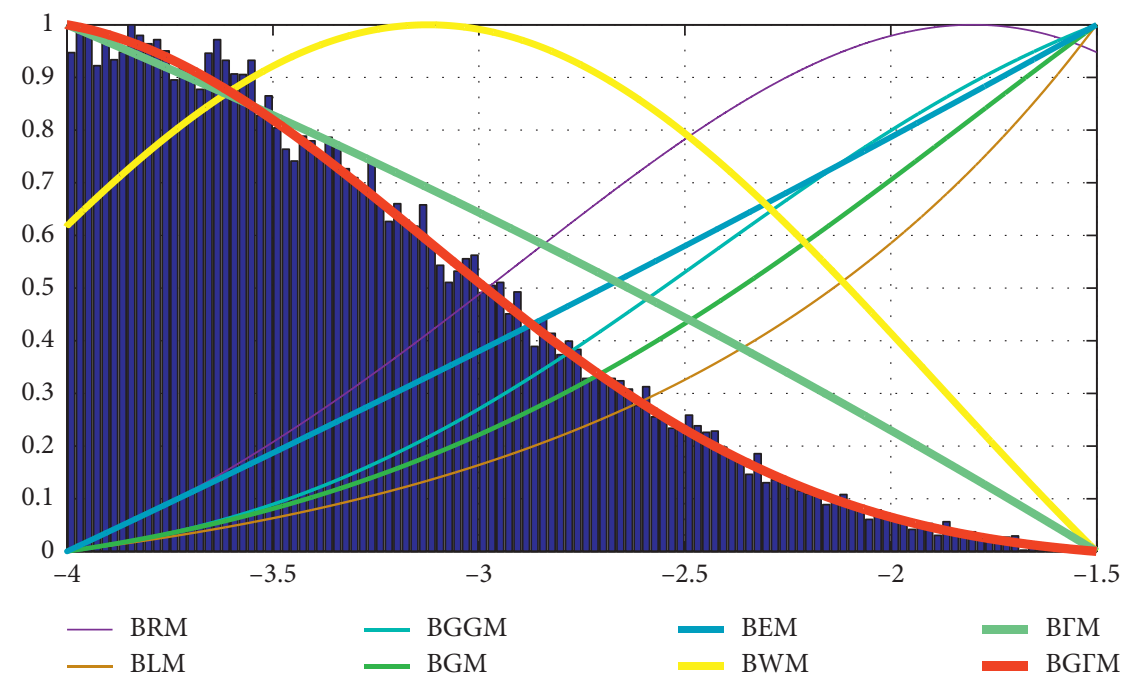

(c)

Figure 6: The estimated histogram. (a) The histogram of the observed data with $u=-0.3 ; \delta=1.5 ; \eta=2, \lambda=3$ and $\beta=0.1$ in the interval $(-4,-1.5)$; (b) the estimated histogram of RM, LM, WM, GM, EM, GGM, ГM, GГM, and GГM; (c) the estimated histogram of BRM, BLM, BWM, BGM, BEM, BGGM, ВГM, and ВGГM. 
TABLE 4: The corresponding $-2 L$ values of models fitted to simulated data in Figures 4-6.

\begin{tabular}{lccc}
\hline Model & Figure 4 & Figure 5 & Figure 6 \\
\hline LM & 340369.6 & 127185.1 & 339937.1 \\
RM & 236056.8 & 58161.5 & 235563.1 \\
GM & 321759.5 & 93056.9 & 321116.7 \\
EM & 342290.4 & 113226.3 & 342259.8 \\
GGM & 328885.3 & 79528 & 327945.2 \\
WM & 172977.9 & 59251.4 & 172863.8 \\
TM & 496904.9 & 99792 & 497023.3 \\
GГM & 199109.3 & 18948.2 & 199442.2 \\
BLM & 157338.1 & 123729.1 & 156760.5 \\
BRM & 132502.2 & 52067.8 & 132867 \\
BGM & 321759.5 & 93056.9 & 321116.7 \\
BEM & 89868.5 & 103830.2 & 90370.9 \\
BGGM & 189787.7 & 59251.4 & 788722.3 \\
BWM & 76569.8 & 78813.4 & 76857.7 \\
BГM & 71019.3 & 63381.9 & 70210 \\
BGГM & 55227.9 & 18944.4 & 55581 \\
\hline
\end{tabular}

Step 3: Check the convergence, if $\left|\partial L(\Theta) / \partial \Theta_{i}\right|_{\Theta=\Theta^{(t)}} \mid$ $<10^{-4}$, for all $1 \leq i \leq 5$ under the constrains $\operatorname{AL}\left(\Theta^{(t)}\right)$ is negative definite, where

$$
\operatorname{AL}(\Theta)=\left(\begin{array}{ccccc}
\frac{\partial^{2} L}{\partial u^{2}} & \frac{\partial^{2} L}{\partial u \partial \delta} & \frac{\partial^{2} L}{\partial u \partial \beta} & \frac{\partial^{2} L}{\partial u \partial \eta} & \frac{\partial^{2} L}{\partial u \partial \lambda} \\
\frac{\partial^{2} L}{\partial u \partial \delta} & \frac{\partial^{2} L}{\partial \delta^{2}} & \frac{\partial^{2} L}{\partial \delta \partial \beta} & \frac{\partial^{2} L}{\partial \delta \partial \eta} & \frac{\partial^{2} L}{\partial \delta \partial \lambda} \\
\frac{\partial^{2} L}{\partial u \partial \beta} & \frac{\partial^{2} L}{\partial \delta \partial \beta} & \frac{\partial^{2} L}{\partial \beta^{2}} & \frac{\partial^{2} L}{\partial \beta \partial \eta} & \frac{\partial^{2} L}{\partial \beta \partial \lambda} \\
\frac{\partial^{2} L}{\partial u \partial \eta} & \frac{\partial^{2} L}{\partial \delta \partial \eta} & \frac{\partial^{2} L}{\partial \beta \partial \eta} & \frac{\partial^{2} L}{\partial \eta^{2}} & \frac{\partial^{2} L}{\partial \eta \partial \lambda} \\
\frac{\partial^{2} L}{\partial u \partial \lambda} & \frac{\partial^{2} L}{\partial \delta \partial \lambda} & \frac{\partial^{2} L}{\partial \beta \partial \lambda} & \frac{\partial^{2} L}{\partial \eta \partial \lambda} & \frac{\partial^{2} L}{\partial \lambda^{2}}
\end{array}\right) .
$$

Then evaluate the function in (29). When the convergence is not verified, then go to step 1 to update the initial point.

Recall that since the matrix $\operatorname{AL}(\Theta)$ be an $5 \times 5$ symmetric matrix and let $A_{k} L(\Theta)$ be the submatrix of $\operatorname{AL}(\Theta)$ obtained by taking the upper left-hand corner $5 \times 5$ submatrix of $\mathrm{AL}(\Theta)$. Furthermore, let $\Delta_{k}=\operatorname{det}\left(A_{k} L(\Theta)\right)$, the $k^{\text {th }}$ principal minor of $\operatorname{AL}(\Theta)$. Then $\operatorname{AL}(\Theta)$ is negative definite if and only if $(-1)^{k} \Delta_{k}>0$ for $k=1,2,3,4,5$. In comparison with the standard EM algorithm, our methodology can make it simple to evaluate the parameters $\beta, \eta$, and $\lambda$ by maximizing the higher bound on the data log-likelihood function as appeared in (42), (49), and (58) separately. In the following section, we will explain the robustness, accuracy, and effectiveness of the proposed model, as compared with other models.

\section{Experiments}

We explain the proposed technique in different examinations. The execution of BGГM is compared with the WM [16], RM [19], EM [14], LM [22], GM [15], GGM [25], ГM [1], GГM [2], BWMM [26], BRM [27], BEM [28], BLM [22], BGM [29, 30], BGGM [22], and ВГM [31]. To measure the fitting precision of every model, we use the corresponding -2 Log-likelihood $(-2 L)$ values of models fitted to data. In general, the smaller values of $(-2 L)$, is the better fit to the data.

5.1. Simulation Study. We generate 40000 random numbers from BGГM with different parameters and bounded support regions see Figures $4-6$. The corresponding $-2 L$ values of models fitted to simulated data are listed in Table 4. We find that ВGГM is the most powerful and has the least $-2 L$. The pdf of BGГM is monotonically increasing, decreasing, bathtub, and upside down bathtub-shaped depending on the parameter values and bounded support regions. So this model is of noticeable importance for image coding and compression applications [32, 33].

\subsection{Real Data Study. We give here six real data as follows:}

(1) The first data set arose in tests on endurance of deep groove ball bearings which is from Lawless (1982, p. 288). The data set is $17.88,28.92,33,41.52,42.12$, $45.6,48.48,51.84,51.96,54.12,55.56,67.80,68.64$, $68.64,68.88,84.12,93.12,98.64,105.12,105.84$, $127.92,128.04,173.40$.

(2) The second data set of the yearly maximum wind speed data in miles/hour, used in this study has been quoted from Castillo (1988) [34].

(3) The third data set of the tensile strength of 100 observations of carbon fibers, the data was obtained from Ref [35]. The data are 3.7, 2.74, 2.73, 2.5, 3.6, 


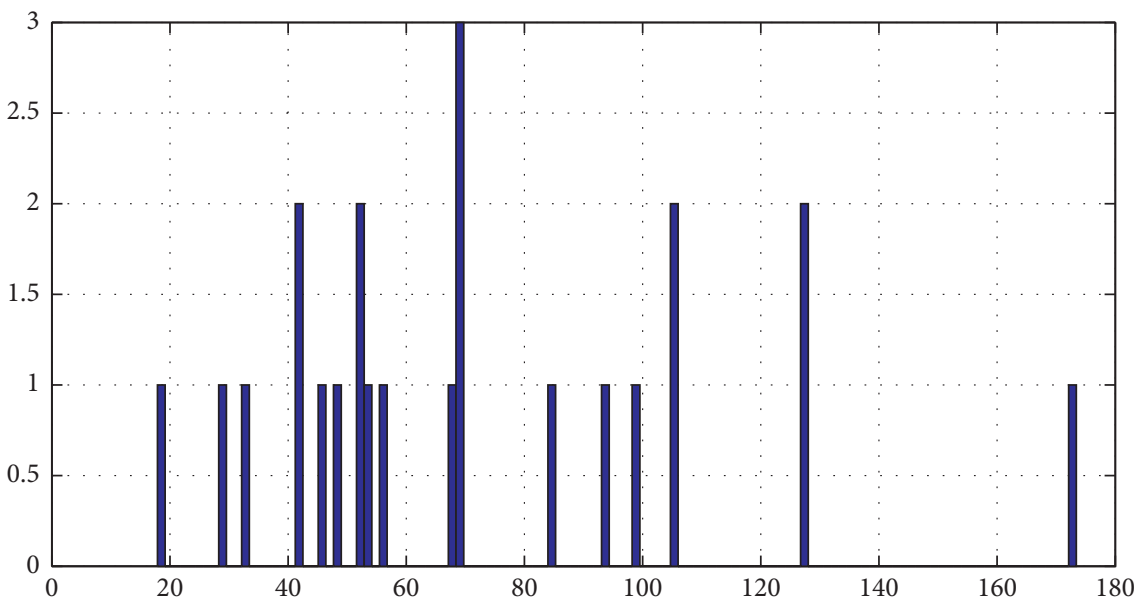

(a)

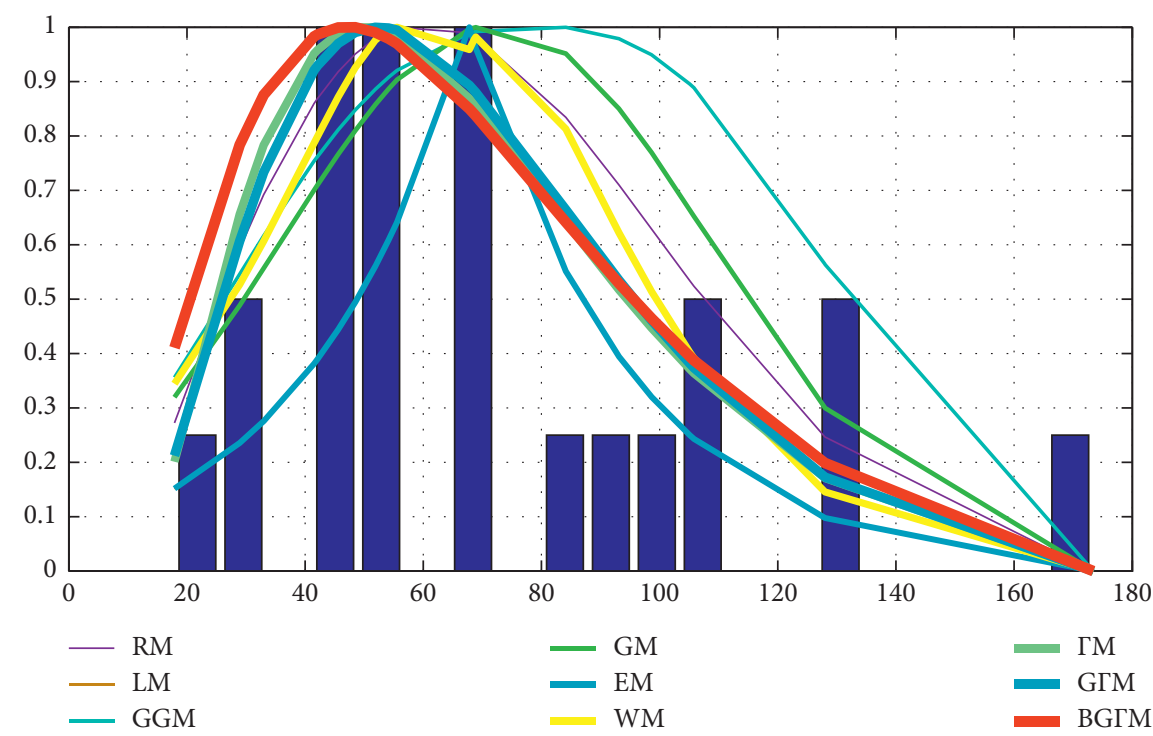

(b)

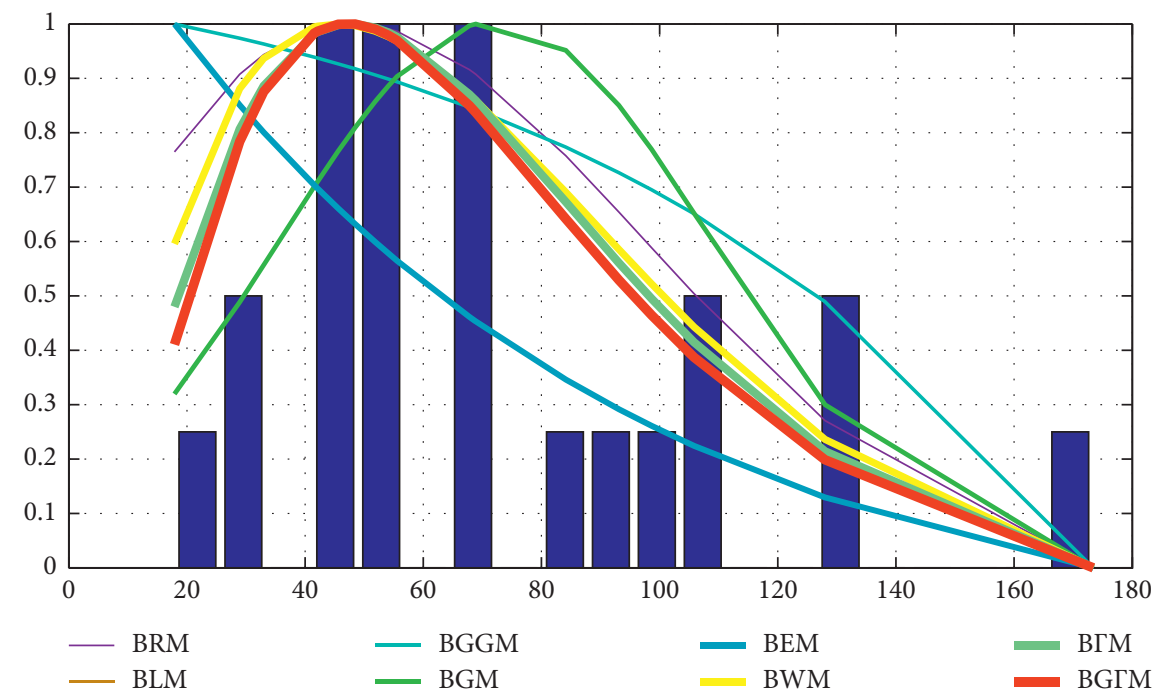

(c)

FIGURE 7: The estimated histogram. (a) The histogram of the tests on endurance of deep groove ball bearings; (b) the estimated histogram of RM, LM, WM, GM, EM, GGM, ГM, GГM, and GГM; (c) the estimated histogram of BRM, BLM, BWM, BGM, ВEM, ВGGM, ВГM, and ВGГM. 


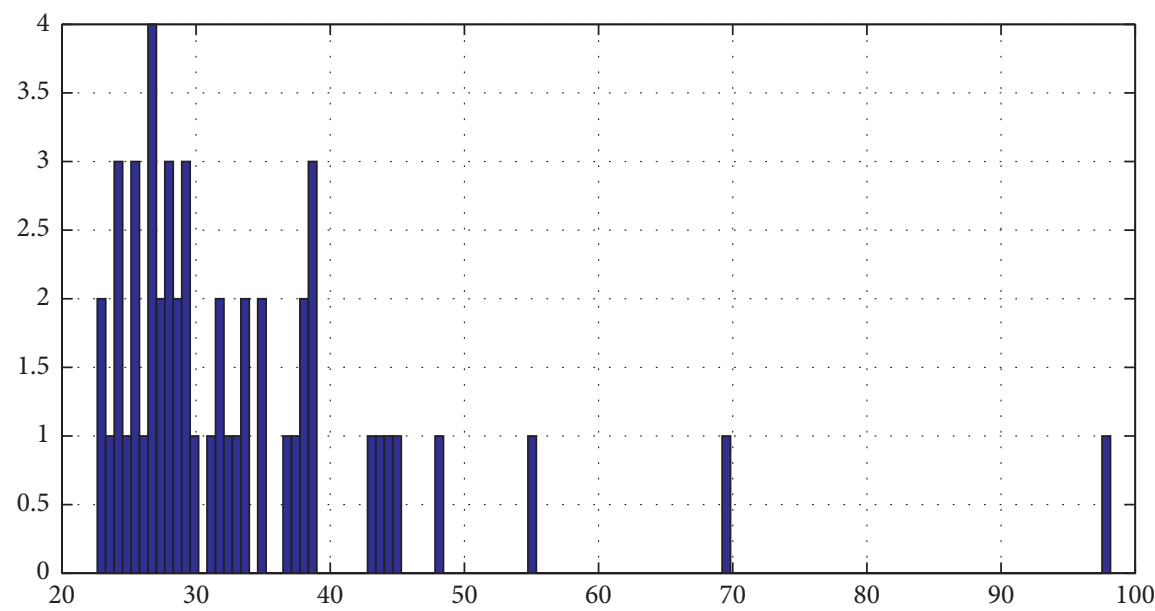

(a)

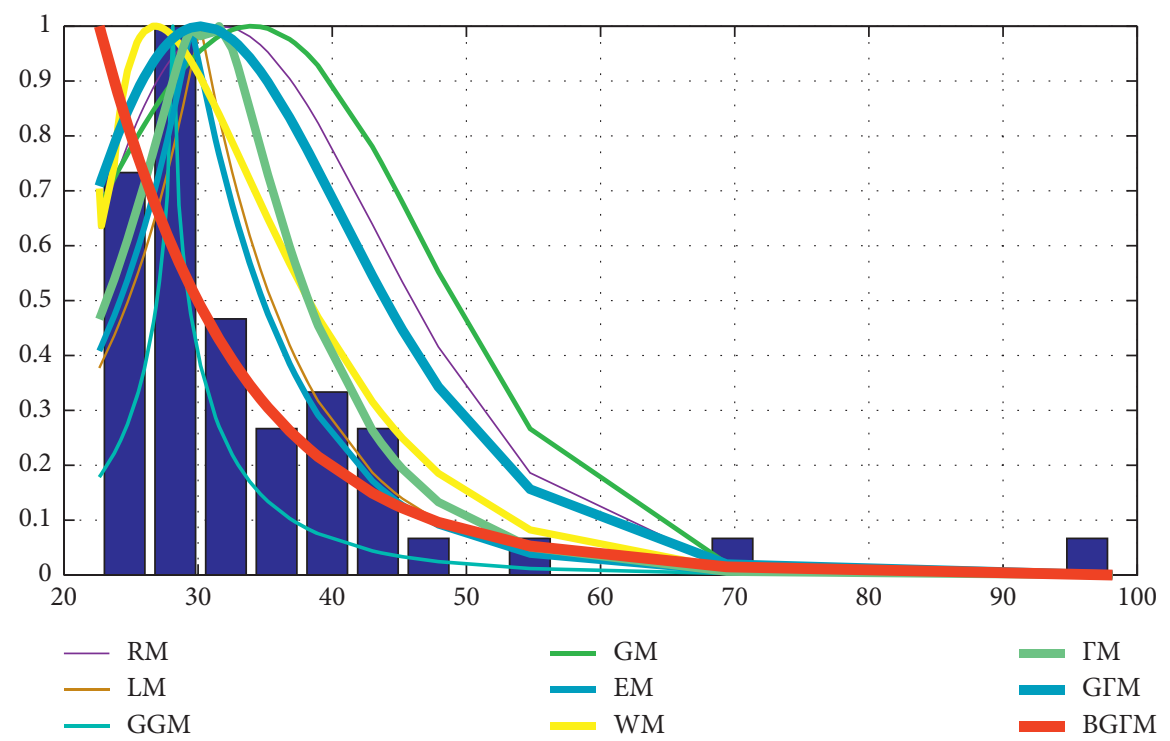

(b)

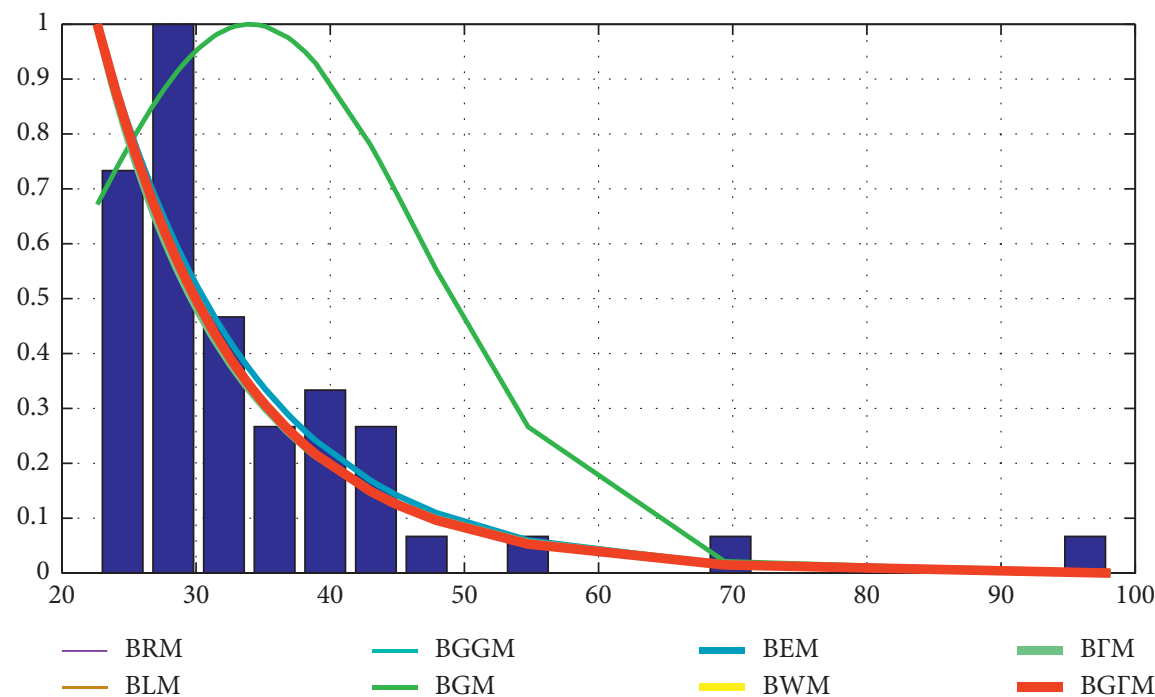

(c)

FIGURE 8: The estimated histogram. (a) The histogram of the yearly maximum wind speed data; (b) the estimated histogram of RM, LM, WM, GM, EM, GGM, ГM, GГM, and GГM; (c) the estimated histogram of BRM, BLM, BWM, BGM, BEM, ВGGM, ВГM, and BGГM. 


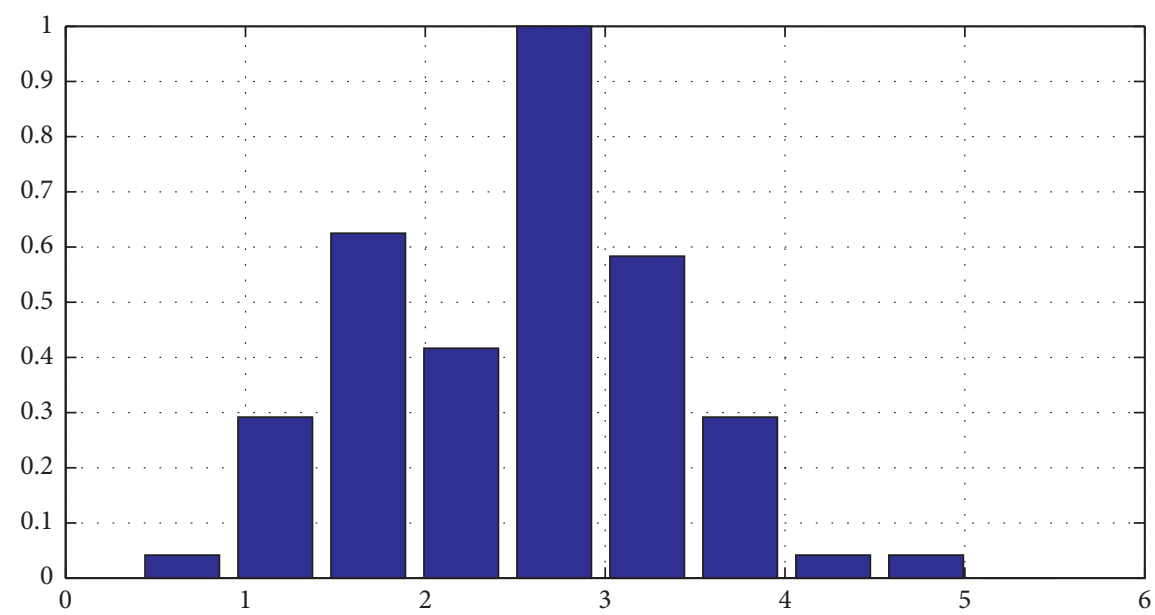

(a)

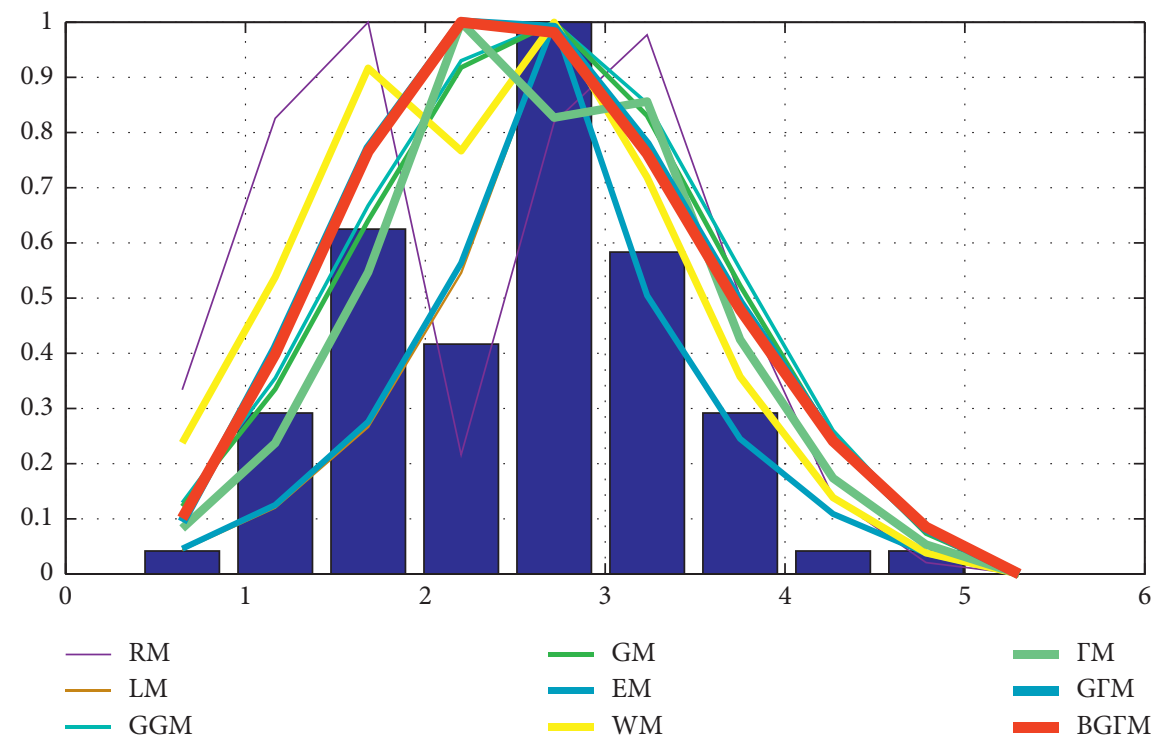

(b)

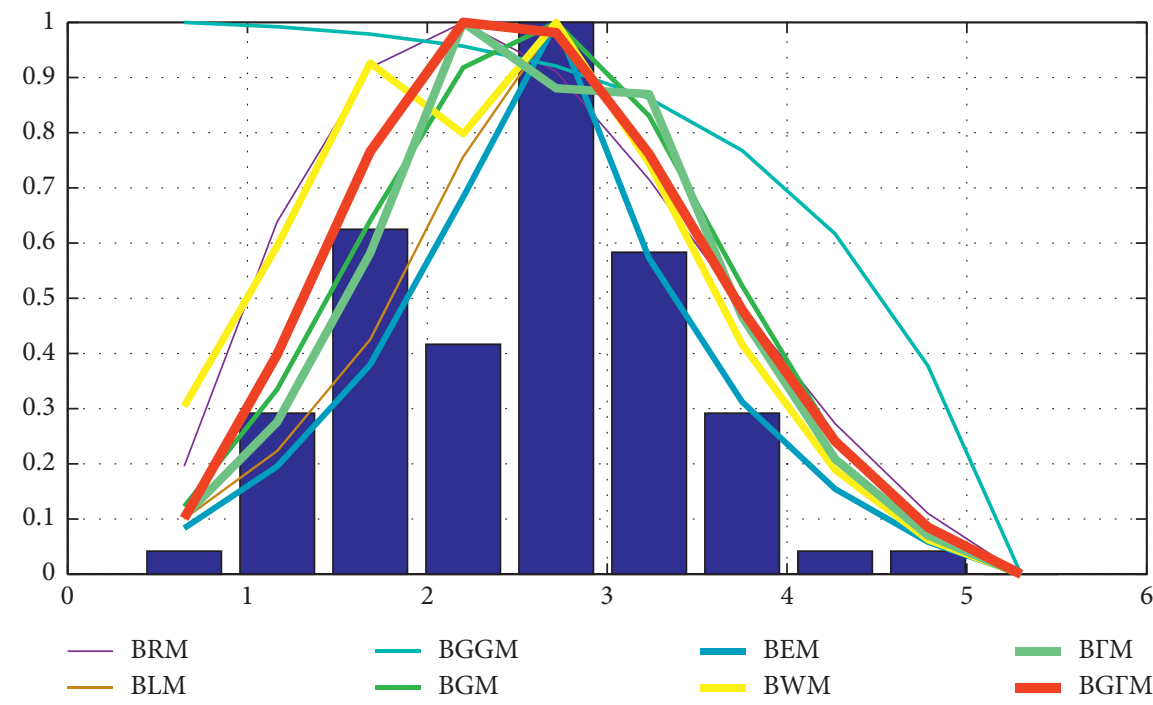

(c)

Figure 9: The estimated histogram. (a) The histogram of the tensile strength of 100 observations of carbon fibers; (b) the estimated histogram of RM, LM, WM, GM, EM, GGM, ГM, GГM, and GГM; (c) the estimated histogram of BRM, BLM, BWM, BGM, BEM, BGGM, ВГM, and ВGГM. 


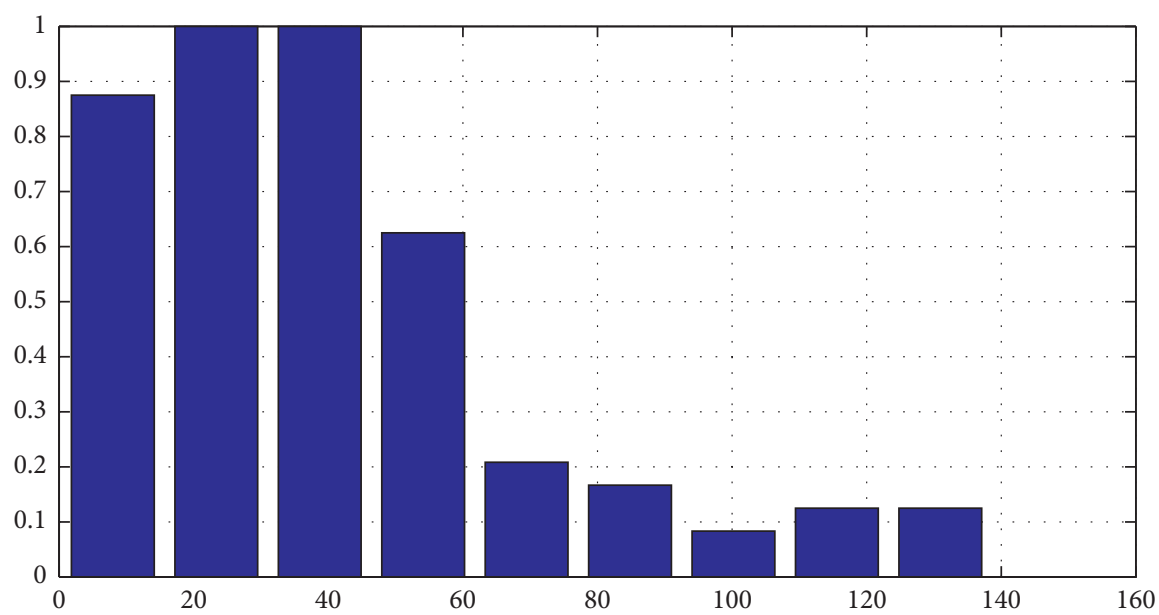

(a)

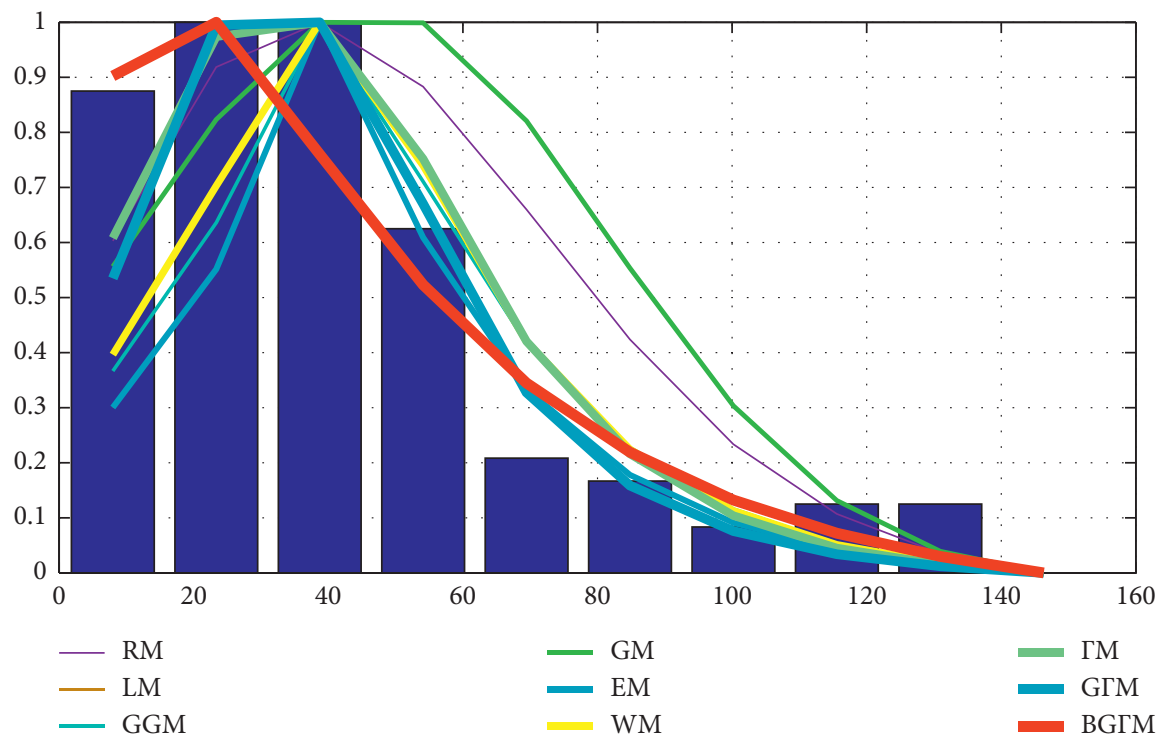

(b)

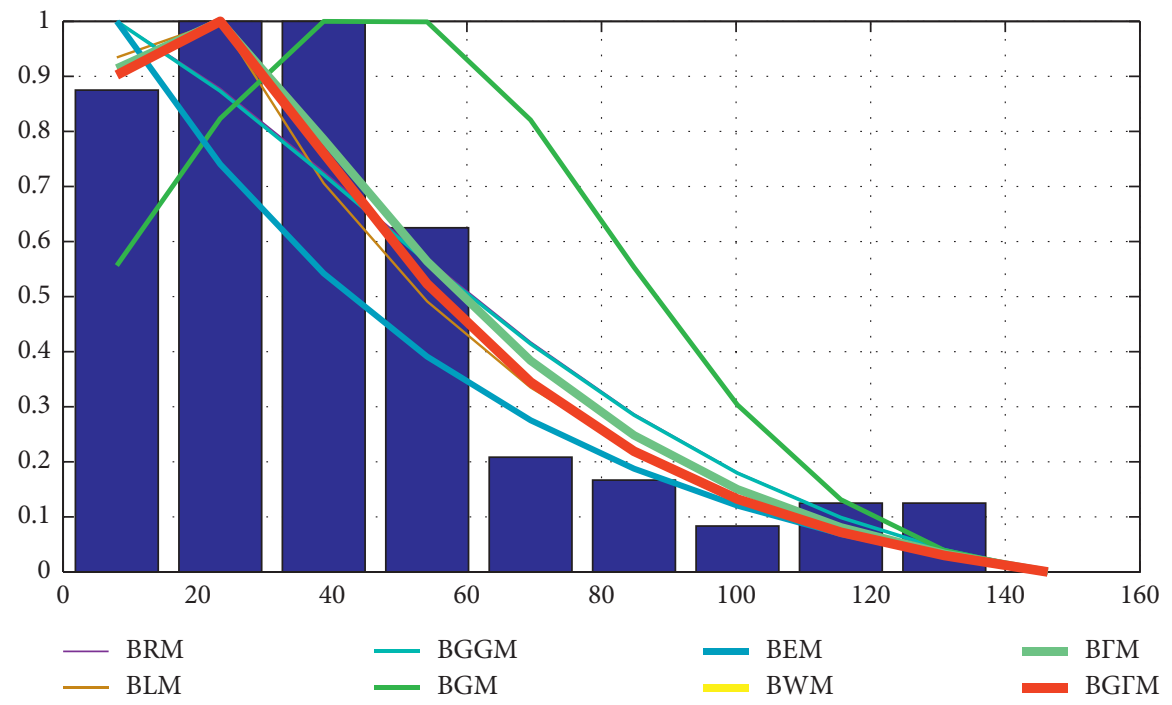

(c)

FIgURE 10: The estimated histogram. (a) The histogram of the survival times of 121 patients with breast cancer; (b) the estimated histogram of RM, LM, WM, GM, EM, GGM, ГM, GГM, and GГM; (c) the estimated histogram of BRM, BLM, BWM, BGM, BEM, BGGM, BГM, and BGГM. 


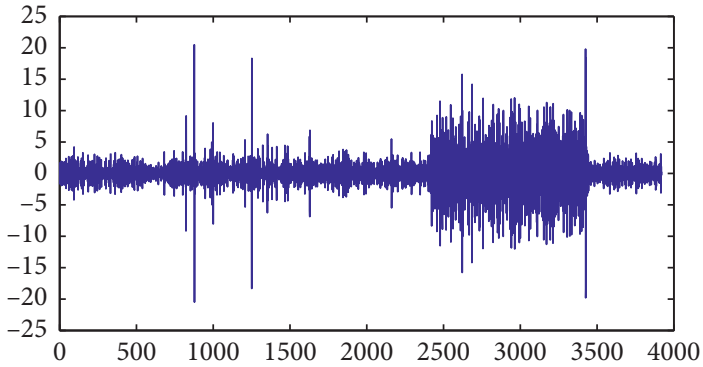

(a)

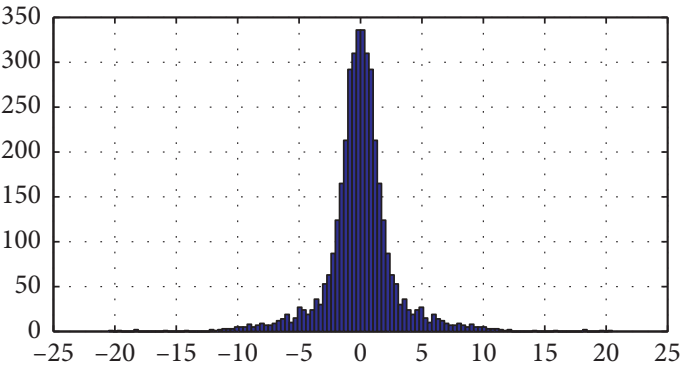

(b)

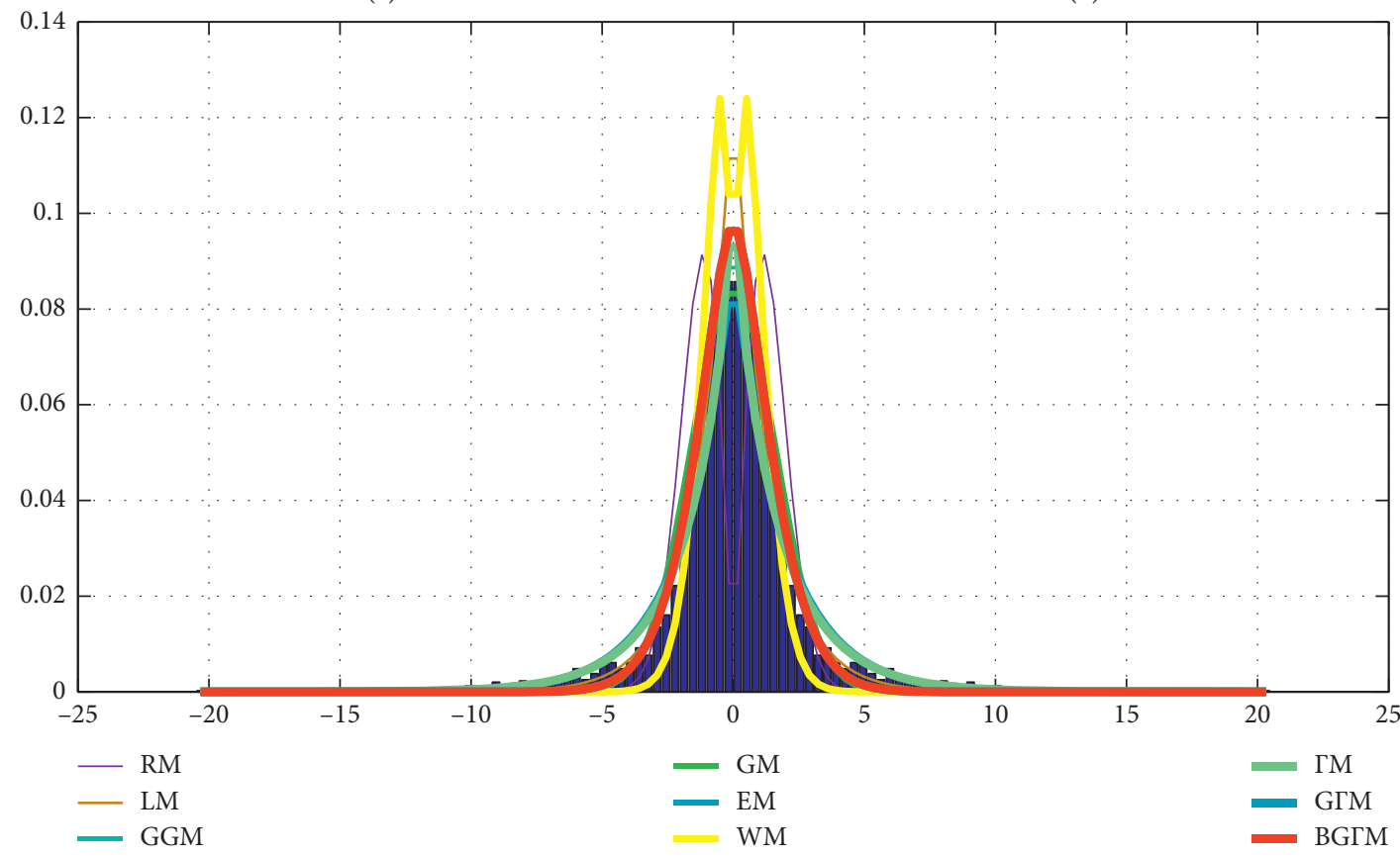

(c)

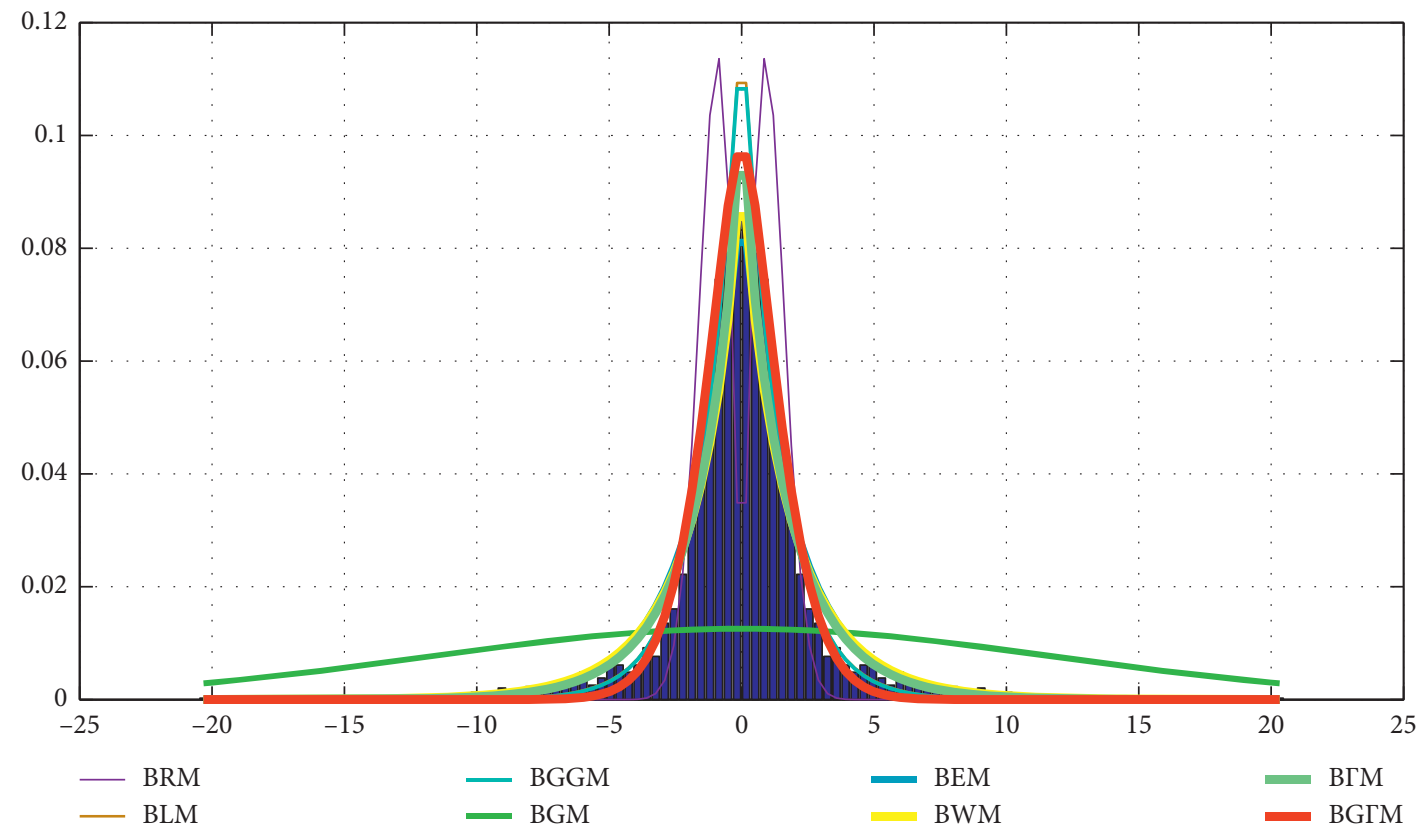

(d)

FIGURE 11: Approximation of the wavelet coefficients. (a) "Leleccum.wav"(leleccum $(1: 3920)$ ); (b) approximation of the wavelet coefficient (db1, CD, level 1) of "leleccum.wav;" (c) the estimated histogram of RM, LM, WM, GM, EM, GGM, ГM, GГM, and GГM; (d) the estimated histogram of BRM, BLM, BWM, BGM, BEM, BGGM, ВГM, and ВGГM. 


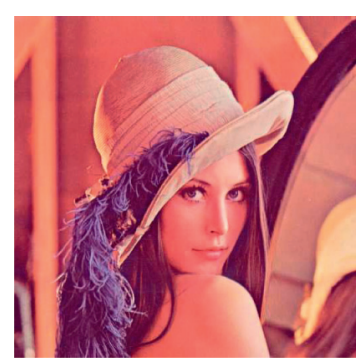

(a)

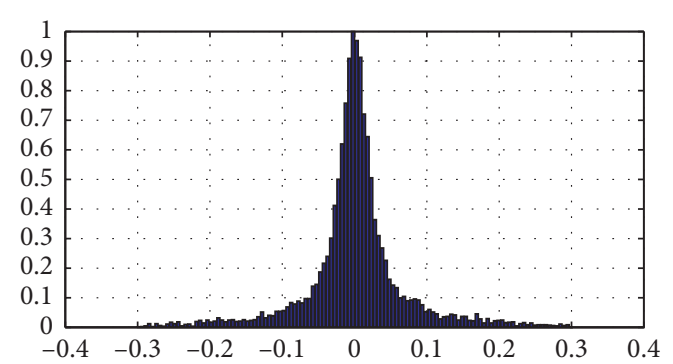

(b)

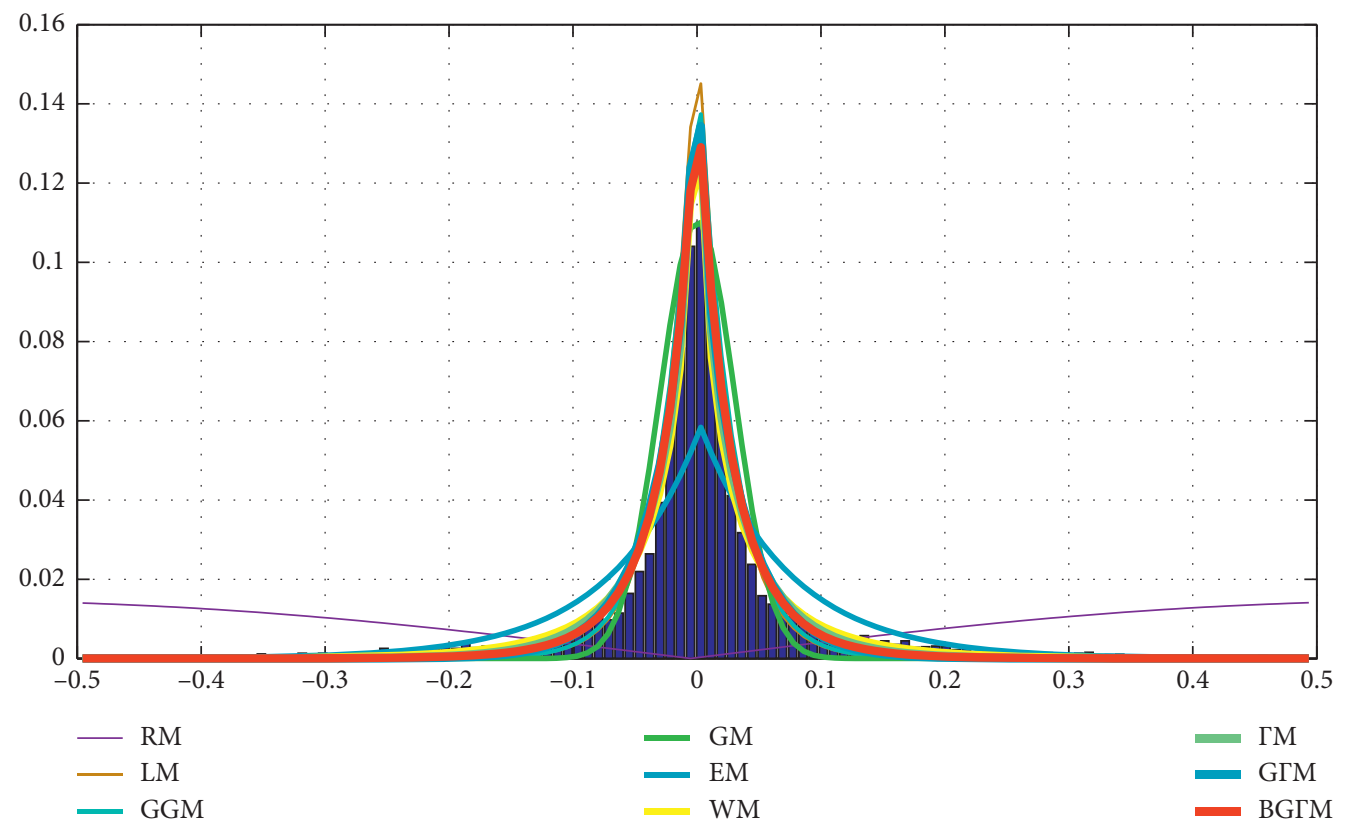

(c)

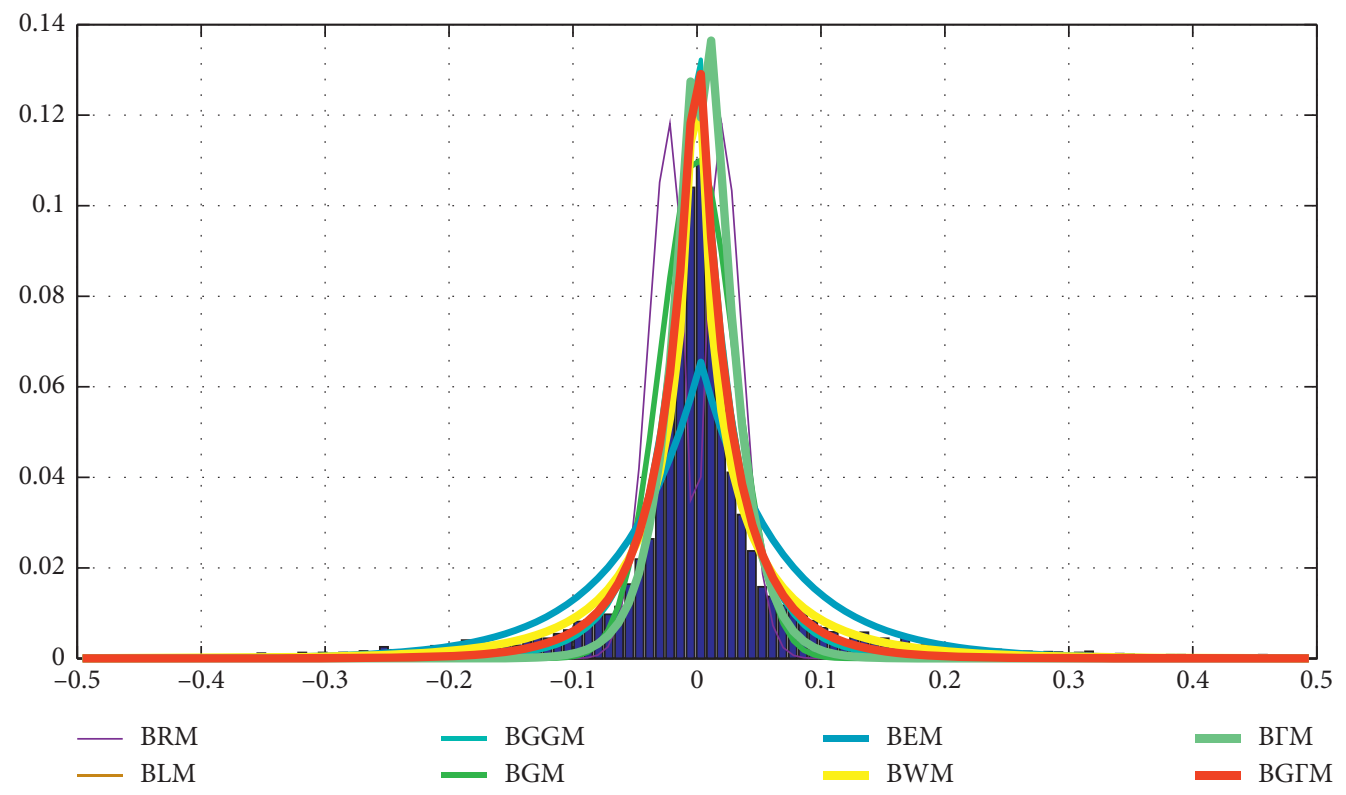

(d)

Figure 12: Approximation of the wavelet coefficients. (a) The original image (lena); (b) the wavelet coefficient (db4, CD, level 1) of lena image in $(-0.5,0.5)$; (c) the estimated histogram of RM, LM, WM, GM, EM, GGM, ГM, GГM, and GГM; (d) the estimated histogram of BRM, BLM, BWM, BGM, BEM, BGGM, ВГM, and ВGГM. 
Table 5: The corresponding $-2 L$ values of models fitted to real data in Figures 7-12.

\begin{tabular}{|c|c|c|c|c|c|c|}
\hline Model & Figure 7 & Figure 8 & Figure 9 & Figure 10 & Figure 11 & Figure 12 \\
\hline LM & 231.689 & 372.636 & 294.201 & 1202.9 & 2054.7969 & 1622.7872 \\
\hline $\mathrm{RM}$ & 258.488 & 445.485 & 311.805 & 1343.8 & 12980.3354 & 20236.904 \\
\hline GM & 230.955 & 396.412 & 285.541 & 1204.7 & 6672.0221 & 10248.145 \\
\hline EM & 231.691 & 372.636 & 294.201 & 1202.9 & 1597.1747 & 1.4026 \\
\hline GGM & 231.743 & 370.125 & 285.727 & 1201.7 & 4469.5735 & 1.6748 \\
\hline WM & 230.41 & 415.379 & 287.88 & 1202.2 & 7595.6214 & 534.9312 \\
\hline$\Gamma \mathrm{M}$ & 305.696 & 373.761 & 314.142 & 1218.7 & 3552.8825 & 739.3194 \\
\hline GГМ & 257.824 & 441.071 & 421.314 & 1198.6 & 1557.8015 & 440.4421 \\
\hline BLM & 225.714 & 343.052 & 282.126 & 1152 & 2020.3745 & 1294.9581 \\
\hline BRM & 224.418 & 343.064 & 285.874 & 1154.8 & 20206.6427 & 20527.3021 \\
\hline BGM & 224.632 & 343.072 & 281.674 & 1155 & 6672.0221 & 10248.145 \\
\hline BEM & 225.714 & 343.052 & 282.098 & 1156.6 & 1597.168 & 881.9213 \\
\hline BGGM & 232.148 & 342.794 & 328.575 & 1154.9 & 2104.6441 & 1361.9667 \\
\hline BWM & 224.164 & 432.44 & 279.486 & 1155.7 & 3551.7368 & 515.5414 \\
\hline ВГМ & 245.613 & 346.724 & 296.563 & 11741.7 & 1557.7918 & 626.7688 \\
\hline ВGГМ & 223.82 & 342.289 & 280.608 & 1151.3 & 1493.2769 & 366.1614 \\
\hline
\end{tabular}

TABLE 6: The corresponding AIC, BIC, and CAIC on real data 4 .

\begin{tabular}{lccc}
\hline Model & AIC & BIC & CAIC \\
\hline BGГM & 1161.308 & 1175.288 & 1161.831 \\
McLL & 1164.661 & 1178.64 & 1165.183 \\
\hline
\end{tabular}

$3.11,3.27,2.87,1.47,3.11,4.42,2.41,3.19,3.22,1.69$, $3.28,3.09,1.87,3.15,4.9,3.75,2.43,2.95,2.97,3.39$, $2.96,2.53,2.67,2.93,3.22,3.39,2.81,4.2,3.33,2.55$, $3.31,3.31,2.85,2.56,3.56,3.15,2.35,2.55,2.59,2.38$, $2.81,2.77,2.17,2.83,1.92,1.41,3.68,2.97,1.36,0.98$, 2.76, 4.91, 3.68, 1.84, 1.59, 3.19, 1.57, 0.81, 5.56, 1.73, $1.59,2,1.22,1.12,1.71,2.17,1.17,5.08,2.48,1.18$, $3.51,2.17,1.69,1.25,4.38,1.84,0.39,3.68,2.48,0.85$, $1.61,2.79,4.7,2.03,1.8,1.57,1.08,2.03,1.61,2.12$, $1.89,2.88,2.82,2.05,3.65$.

(4) The fourth data set of the survival times of 121 patients with breast cancer, the data was obtained from Ref [36].

(5) In this part "Leleccum.wav"(leleccum $(1: 3920))$ is disintegrated into three high-pass subbands $(\mathrm{CH}$, $\mathrm{CV}, \mathrm{CD}$ ) and one low-pass subband (CA). The Daubechies channel bank (db1) is used. The fifth data set is the approximation of the wavelet coefficient (db1, CD, level 1) of "leleccum.wav" in the interval $(-20.32,20.32)$.

(6) The wavelet approximation coefficient is an essential issue in computer vision as it assumes an important part in an extensive range of applications. The image of (lena) is decomposed into three high-pass subbands $(\mathrm{CH}, \mathrm{CV}, \mathrm{CD})$ and one low-pass subband (CA). The Daubechies filter bank (db4) is used. The sixth data set is the wavelet coefficients of the highpass subband (CD), level 1 in the interval $(-0.5,0.5)$.

The histogram for all real sets and their estimated pdfs for the fitted models are displayed in Figure 7-12. The corresponding $-2 L$ values of models fitted to real data are listed in Table 5. Therefore, the proposed model provides a better fit to these data and has the least $-2 L$.Secondly, if we compare the power of our model with modified generalized gamma distribution (MGG) having 6-parameters defined and studied in [12] on real data 3 , we have $-2 L=280.608$ and $-2 L=282.692$, respectively. Hence, ВGГM is high flexible than MGG for this data. Furthermore, we compare McDonald log-logistic distribution (McLL) [36] with our model BGГM. The model selection is carried out using the following statistics: AIC (Akaike information criterion), CAIC (consistent Akaike information criterion), and BIC (Bayesian information criterion). The corresponding values of models fitted to real data 4 are listed in Table 6 . We find that BGГM is more flexible than McLL in this case.

\section{Conclusions}

A bounded generalized Gamma model with five parameters, whose hazard function can be monotonically increasing, decreasing, bathtub, and upside down bathtub-shaped depending on the parameter values, has been introduced and studied. Some mathematical and statistical properties of the new model are investigated. We estimate the model parameters using maximum log-likelihood function and find a closed form of some parameters by the Newton Raphson method. The predictive ability of our model is found to be comparable or superior to widely accepted distributions. The performance of the model has the smallest $-2 L$ values. A simulation study was carried out to evaluate the predictive ability of our model to fit any kind of data with bounded support regions and compare it with other distributions. The power of the new model is illustrated by means of application to six real data sets. The BGГM performs significantly better than the others distributions when sample sizes are 
small. Thus, it is less affected by sample size and is more robust. Also the accuracy of the proposed model for wavelet histogram fitting of image and sound is high. We hope that this model may attract wider applications on the modeling of the probability density function of the data via ВGГD in video coding and image denoising as a future work.

\section{Data Availability}

The data used to support the findings of this study are available from the corresponding author upon request.

\section{Ethical Approval}

This article does not contain any studies with human participants or animals performed by any of the authors.

\section{Conflicts of Interest}

The authors declare that they have no competing interests.

\section{Authors' Contributions}

All the authors contributed equally to the writing of this paper. All authors read and approved the final manuscript.

\section{References}

[1] J. M. Nicolas and F. Tupin, "Gamma mixture modeled with second kind statistics: application to SAR image processing," in Proceedings of the IGARSS, pp. 2489-2491, Toronto, Canada, June 2002.

[2] E. W. Stacy, "A generalization of the gamma distribution," The Annals of Mathematical Statistics, vol. 33, no. 3, pp. 11871192, 1962.

[3] L. Amoroso, "Ricerche intorno alla curva dei redditi," Annali di Matematica Pura ed Applicata, vol. 2, no. 1, pp. 123-159, 1925.

[4] N. L. Johnson, S. Kotz, and N. Balakrishnan, Continuous Univariate Distributions, Vol. 1, Wiley and Sons, Inc., New York, NY, USA, 2nd edition, 1994.

[5] G. S. Mudholkar and D. K. Srivastava, "Exponentiated Weibull family for analyzing bathtub failure-rate data," IEEE Transactions on Reliability, vol. 42, no. 2, pp. 299-302, 1993.

[6] S. K. Agarwal and J. A. Al-Saleh, "Generalized gamma type distribution and its hazard rate function," Communications in Statistics - Theory and Methods, vol. 30, no. 2, pp. 309-318, 2001.

[7] N. Balakrishnan and Y. Peng, "Generalized gamma frailty model," Statistics in Medicine, vol. 25, no. 16, pp. 2797-2816, 2006.

[8] G. M. Cordeiro, E. M. M. Ortega, and G. O. Silva, "The exponentiated generalized gamma distribution with application to lifetime data," Journal of Statistical Computation and Simulation, vol. 81, no. 7, pp. 827-842, 2012.

[9] S. Nadarajah and A. K. Gupta, "A generalized gamma distribution with application to drought data," Mathematics and Computers in Simulation, vol. 74, no. 1, pp. 1-7, 2007.

[10] L. Chen, V. Singh, and F. Xiong, "An entropy-based generalized gamma distribution for flood frequency analysis," Entropy, vol. 19, no. 6, p. 239, 2017.
[11] Z. Zhao, L. Guo, M. Jia, and L. Wang, "The generalized gamma-DBN for high-resolution SAR image classification," Remote Sensing, vol. 10, no. 6, p. 878, 2018.

[12] M. Mead, M. Nassar, and S. Dey, "A generalization of generalized gamma distributions," Pakistan Journal of Statistics and Operation Research, vol. 1, no. 14, pp. 121-138, 2018.

[13] D. Fulger, E. Scalas, and G. Germano, "Random numbers from the tails of probability distributions using the transformation method," Fractional Calculus and Applied Analysis, vol. 16, no. 2, pp. 332-353, 2013.

[14] A. Feldmann and W. Whitt, "Fitting mixtures of exponentials to long-tail distributions to analyze network performance models," Performance Evaluation, vol. 31, no. 3-4, pp. 245-279, 1998.

[15] G. McLachlan and D. Peel, Finite Mixture Models, John Wiley \& Sons, New York, NY, USA, 2000.

[16] L. Attardi, M. Guida, and G. Pulcini, "A mixed-Weibull regression model for the analysis of automotive warranty data," Reliability Engineering \& System Safety, vol. 87, no. 2, pp. 265-273, 2005.

[17] T. Bučar, D. M. Nago, and M. jdiga, "Reliability approximation using finite Weibull mixture distributions," Reliability Engineering \& System Safety, vol. 84, pp. 241-251, 2004.

[18] J. A. Carta and P. Ramírez, "Analysis of two-component mixture Weibull statistics for estimation of wind speed distributions," Renewable Energy, vol. 32, no. 3, pp. 518-531, 2007.

[19] K. V. Sorensen and S. V. Andersen, "Rayleigh mixture modelbased hidden markov modeling and estimation of noise in noisy speech signals," IEEE Transactions on Audio Speech and Language Processing, vol. 15, no. 3, pp. 901-917, 2007.

[20] J. Seabra, F. Ciompi, P. Radeva, and J. Miguel Sanches, "A Rayleigh mixture model for IVUS imaging," Ultrasound Imaging. Advances and Applications, Springer-Verlag New York, New York, NY, USA, 2012.

[21] M. N. Do and M. Vetterli, "Wavelet-based texture retrieval using generalized Gaussian density and Kullback-Leibler distance," IEEE Transactions on Image Processing, vol. 11, no. 2, pp. 146-158, 2002.

[22] T. M. Nguyen, Q. M. Jonathan Wu, and H. Zhang, "Bounded generalized Gaussian mixture model," Pattern Recognition, vol. 47, no. 9, pp. 3132-3142, 2014.

[23] C. M. Bishop, Pattern Recognition and Machine Learning, Springer, Berlin, Germany, 2006.

[24] P. J. Huber, Robust Statistics, Vol. 43, Wiley, New York, NY, USA, 1981.

[25] M. Allili, "Wavelet modeling using finite mixtures of generalized Gaussian distributions: application to texture discrimination and retrieval," IEEE Transactions on Image Processing, vol. 21, no. 4, pp. 1452-1464, 2012.

[26] T. Zhang and M. Xie, "On the upper truncated Weibull distribution and its reliability implications," Reliability Engineering \& System Safety, vol. 96, no. 1, pp. 194-200, 2011.

[27] A. R. Sudamani Ramaswamy and S. Jayasri, "Time truncated chain sampling plans for generalized Rayleigh distribution," International Refereed Journal of Engineering and Science (IRJES), vol. 3, no. 2, pp. 49-53, 2014.

[28] M. Raschke, "Inference for the truncated exponential distribution," Stochastic Environmental Research and Risk Assessment, vol. 26, no. 1, pp. 127-138, 2012.

[29] P. Hedelin and J. Skoglund, "Vector quantization based on Gaussian mixture models," IEEE Transactions on Speech and Audio Processing, vol. 8, no. 4, pp. 385-401, 2000. 
[30] J. Lindblom and J. Samuelsson, "Bounded support Gaussian mixture modeling of speech spectra," IEEE Transactions on Speech and Audio Processing, vol. 11, no. 1, pp. 88-99, 2003.

[31] C. S. Coffey and K. E. Muller, "Properties of doubly-truncated gamma variables," Communications in Statistics-Theory and Methods, vol. 29, no. 4, pp. 851-857, 2000.

[32] S. G. Chang, B. Bin Yu, and M. Vetterli, "Adaptive wavelet thresholding for image denoising and compression," IEEE Transactions on Image Processing, vol. 9, no. 9, pp. 1532-1546, 2000.

[33] S. Kasaei, M. Deriche, and B. Boashash, "A novel fingerprint image compression technique using wavelets packets and pyramid lattice vector quantization," IEEE Transactions on Image Processing, vol. 11, no. 12, pp. 1365-1378, 2002.

[34] E. Castillo, Extreme Value Theory In Engineering, Vol. 334, Academic Press, London, UK, 1988.

[35] D. F. Andrews and A. M. Herzberg, Data: A Collection of Problems from Many Fields for the Student and Research Worker, Springer Series in Statistics, New York, NY, USA, 1985.

[36] M. H. Tahir, M. Mansoor, M. Zubair, and G. G. Hamedani, "McDonald log-logistic distribution with an application to breast cancer data," Journal of Statistical Theory and Applications, vol. 1, no. 13, pp. 65-82, 2014. 